\title{
Présence des hydrocarbures aromatiques polycycliques (HAP) dans les compartiments biotiques et abiotiques de la rivière et du fjord du Saguenay Presence of polycyclic aromatic hydrocarbons (PAH) in biotic
and abiotic compartments of the Saguenay river and fjord.
}

\author{
Émilien Pelletier, Isabelle Desbiens, Peggy Sargian, Nathalie Côté, Antonio
} Curtosi et Richard St-Louis

Volume 22, numéro 2, 2009

URI : https://id.erudit.org/iderudit/037483ar

DOI : https://doi.org/10.7202/037483ar

Aller au sommaire du numéro

Éditeur(s)

Université du Québec - INRS-Eau, Terre et Environnement (INRS-ETE)

ISSN

1718-8598 (numérique)

Découvrir la revue

Citer cet article

Pelletier, É., Desbiens, I., Sargian, P., Côté, N., Curtosi, A. \& St-Louis, R. (2009). Présence des hydrocarbures aromatiques polycycliques (HAP) dans les compartiments biotiques et abiotiques de la rivière et du fjord du Saguenay. Revue des sciences de l'eau / Journal of Water Science, 22(2), 235-251. https://doi.org/10.7202/037483ar

\section{Résumé de l'article}

Ce document présente une réévaluation approfondie de la présence des hydrocarbures aromatiques polycycliques (HAP) dans la rivière et le fjord du Saguenay, un système aquatique fortement affecté par les activités anthropiques et notamment par les effluents provenant des alumineries entre les années 1950 et 1980. La matière particulaire en suspension (MPS), les sédiments de surface ainsi que certains organismes benthiques, échantillonnés en 2002 et 2003, ont été analysés pour leur contenu en HAP. Les résultats montrent qu'il n’y a pas de différence significative dans la distribution des HAP extraits de la MPS échantillonnée dans les parties supérieure et inférieure de la rivière Saguenay. Cependant, une variation saisonnière des HAP particulaires totaux apparaît avec des concentrations variant de $12 \mu \mathrm{g} \cdot \mathrm{g}^{-1}$ en hiver à $3,2 \mu g^{\circ} \mathrm{g}^{-1}$ en été. Nous avons estimé que la contribution en HAP particulaires de la rivière Saguenay vers le fjord était d'environ $606 \mathrm{~kg}$ par année. Un examen détaillé des profils chromatographiques des HAP nous montre une prédominance des hydrocarbures légers (2 et 3 cycles) dans la MPS de la rivière par opposition aux HAP plus lourds ( 4 et 5 cycles) surtout présents dans les sédiments de surface du fjord. Le niveau de contamination observé dans les sédiments de surface du fjord du Saguenay en 2002 a été comparé à celui rapporté pour des sédiments échantillonnés en 1982. Toutes les carottes de sédiments montrent des concentrations maximales des HAP dans une couche située entre 15 et $28 \mathrm{~cm}$ de profondeur indiquant un enfouissement lent mais constant des sédiments fortement contaminés apportés au fjord dans les années 1960 et 1970. Les HAP totaux mesurés dans nos échantillons de 2002 sont de 8 à 30 fois inférieurs aux concentrations mesurées en 1982. Les HAP mesurés dans les tissus biologiques de quelques espèces benthiques et épi-benthiques du fjord montrent une prédominance des HAP légers (2 à 3 cycles) avec les plus hautes valeurs présentes chez le vers Nereis sp. et la crevette Pandalus borealis de la baie des Ha! Ha! Avec la réduction importante des sources industrielles des hydrocarbures aromatiques, les hydrocarbures pétrogéniques représentent aujourd'hui une importante contribution à la contamination globale du système Saguenay.
Ce document est protégé par la loi sur le droit d'auteur. L’utilisation des services d'Érudit (y compris la reproduction) est assujettie à sa politique d'utilisation que vous pouvez consulter en ligne.

https://apropos.erudit.org/fr/usagers/politique-dutilisation/ 


\title{
PRÉSENCE DES HYDROCARBURES AROMATIQUES POLYCYCLIQUES (HAP) DANS LES COMPARTIMENTS BIOTIQUES ET ABIOTIQUES DE LA RIVIÈRE ET DU FJORD DU SAGUENAY*
}

Presence of polycyclic aromatic bydrocarbons $(\mathrm{PAH})$ in biotic and abiotic compartments of the Saguenay river and fjord.

\author{
Émilien PELleTIER ${ }^{1 *}$, Isabelle DeSBIENS ${ }^{1}$, Peggy SARgIAN ${ }^{1}$, Nathalie CÔTÉ $^{1}$, \\ ANTONIO CURTOSI ${ }^{1}$ ET RichaRd ST-LOUIS ${ }^{1}$ \\ ${ }^{1}$ Institut des sciences de la mer de Rimouski (ISMER), Université du Québec à Rimouski, \\ 310, allée des Ursulines, Rimouski, Québec, Canada G5L 3A1
}

Reçu le 28 août 2008, accepté le 15 novembre 2008

\section{RÉSUMÉ}

Ce document présente une réévaluation approfondie de la présence des hydrocarbures aromatiques polycycliques (HAP) dans la rivière et le fjord du Saguenay, un système aquatique fortement affecté par les activités anthropiques et notamment par les effluents provenant des alumineries entre les années 1950 et 1980. La matière particulaire en suspension (MPS), les sédiments de surface ainsi que certains organismes benthiques, échantillonnés en 2002 et 2003, ont été analysés pour leur contenu en HAP. Les résultats montrent qu'il n'y a pas de différence significative dans la distribution des HAP extraits de la MPS échantillonnée dans les parties supérieure et inférieure de la rivière Saguenay. Cependant, une variation saisonnière des HAP particulaires totaux apparaît avec des concentrations variant de $12 \mu \mathrm{g} \bullet \mathrm{g}^{-1}$ en hiver à $3,2 \mu \mathrm{g} \bullet \mathrm{g}^{-1}$ en été. Nous avons estimé que la contribution en HAP particulaires de la rivière Saguenay vers le ford était d'environ $606 \mathrm{~kg}$ par année. Un examen détaillé des profils chromatographiques des HAP nous montre une prédominance des hydrocarbures légers ( 2 et 3 cycles) dans la MPS de la rivière par opposition aux HAP plus lourds ( 4 et 5 cycles) surtout présents dans les sédiments de surface du fjord. Le niveau de contamination observé dans les sédiments de surface du fjord du Saguenay en 2002 a été comparé à celui rapporté pour des sédiments échantillonnés en 1982. Toutes les carottes de sédiments montrent des concentrations maximales des HAP dans une couche située entre 15 et $28 \mathrm{~cm}$ de profondeur indiquant un enfouissement lent mais constant des sédiments fortement contaminés apportés au fjord dans les années 1960 et 1970. Les HAP

* Parc marin Saguenay-Saint-Laurent

*Auteur pour correspondance :

Téléphone: $418723-1986$ poste 176

Télécopie: 418 724-1842

Courriel : emilien_pelletier@uqar.qc.ca 
totaux mesurés dans nos échantillons de 2002 sont de 8 à 30 fois inférieurs aux concentrations mesurées en 1982. Les HAP mesurés dans les tissus biologiques de quelques espèces benthiques et épi-benthiques du fjord montrent une prédominance des HAP légers ( 2 à 3 cycles) avec les plus hautes valeurs présentes chez le vers Nereis sp. et la crevette Pandalus borealis de la baie des $\mathrm{Ha}$ ! Ha! Avec la réduction importante des sources industrielles des hydrocarbures aromatiques, les hydrocarbures pétrogéniques représentent aujourd'hui une importante contribution à la contamination globale du système Saguenay.

Mots clés : Hydrocarbures aromatiques polycycliques, évolution temporelle, Fjord du Saguenay, matériel particulaire en suspension, sédiment de surface, métabolites, invertébrés.

\section{ABSTRACT}

This paper presents an exhaustive reevaluation of the presence of polycyclic aromatic hydrocarbons (PAHs) in the Saguenay Fjord and River, an aquatic environment strongly affected by anthropogenic activities and particularly by industrial effluents from aluminum plants in the years 1950 to 1980 . Suspended particulate matter (SPM), surface sediment, and a number of organisms have been sampled in 2002 and 2003 and analyzed for their content in PAHs. Results indicate that distribution of PAHs extracted from SPM sampled in the upper section of the river is not statistically different from the distribution observed for particles from the lower section of the river. However, a seasonal change was observed in total particulate PAHs with concentrations ranging from $12 \mu{\mathrm{g} \bullet \mathrm{g}^{-1}}^{1}$ in winter to $3.2 \mu \mathrm{g} \mathrm{g}^{-1}$ in summer. The contribution of particulate $\mathrm{PAHs}$ from the river to the fjord was estimated to $606 \mathrm{~kg}$ per year. A detailed examination of PAH chromatograms revealed the predominance of light hydrocarbons (2 and 3 rings) in river SPM in contrast to heavier hydrocarbons ( 4 and 5 rings) particularly abundant in surface sediment from the fjord. The contamination level observed in surface sediments of the Saguenay Fjord in 2002 has been compared to previously published data obtained for samples obtained at the same locations in 1982. All PAH profiles showed maximum concentrations in a layer between 15 and $28 \mathrm{~cm}$ depth, indicating a slow but constant burying process of highly contaminated sediment brought to the fjord in the years 1960 to 1970 . Total PAHs determined in our samples (2-3 cm layer) are 8 to 30 times lower than those reported for samples collected in 1982. PAHs determined in biological tissues of benthic and epi-benthic species indicated the predominance of light PAHs (2 to 3 rings) with higher values found in shrimps and worms in the Baie des Ha! Ha! With the reduction of industrial sources of pyrogenic hydrocarbons, petrogenic hydrocarbons account now for a major contribution to the global contamination of the Saguenay system.

\section{Keywords: polycyclic aromatic hydrocarbons, temporal changes, Saguenay Fjord, suspended particulate matter, surface sediment, metabolites, invertebrates.}

\section{INTRODUCTION}

Les hydrocarbures aromatiques polycycliques (HAP) constituent une famille de composés distribués de façon ubiquiste dans les environnements dulcicoles et marins et provenant de sources naturelles et anthropiques diverses (BATES et al., 1987; DOONG et LIN, 2004; FERNANDES et al., 1999; YUNKER et al., 2002). Quelques HAP possèdent des propriétés cancérigènes, tératogènes et mutagènes qui amènent des dangers pour les organismes aquatiques et les humains (STRAF et al., 2005; WHITE et al., 1997). La majorité des HAP présente un fort caractère hydrophobe et tend à s'adsorber aux particules organiques en suspension et à s'accumuler dans les sédiments et les sols particulièrement pour les composés lourds possédant une très faible solubilité dans l'eau (GSCHWEND et HITES, 1981; MACKAY et al., 1992; STARK et al., 2003). Il en résulte une persistance à long terme des HAP dans les sédiments côtiers qui présentent alors une menace toxique pour les organismes benthiques puisque leur forte hydrophobicité promeut leur bioaccumulation dans les tissus biologiques (VARANASI et STEIN, 1991).

La rivière et le fjord du Saguenay constituent un système hydrogéologique d'une longueur de $167 \mathrm{~km}$, ayant un bassin versant de $1,7 \times 104 \mathrm{~km}^{2}$ avec un débit moyen de $2100 \mathrm{~m}^{3} \bullet \mathrm{s}^{-1}$ à son embouchure à Tadoussac (FORTIN et PELLETIER, 1995), ce qui en fait le principal tributaire du Saint-Laurent. Les derniers $105 \mathrm{~km}$ du système forment le fjord proprement dit, dont la profondeur peut atteindre $275 \mathrm{~m}$ dans le bassin central avec un seuil d'à peine $20 \mathrm{~m}$ de profondeur à la jonction avec l'estuaire du Saint-Laurent. La colonne d'eau du fjord est caractérisée par une brusque variation de la salinité et de la température dans les dix premiers mètres (sauf en hiver) délimitant une couche de surface saumâtre et plus chaude provenant de l'amont et une couche profonde froide et salée provenant des eaux intermédiaires de l'estuaire maritime du Saint-Laurent (DRAINVILLE, 1968). Au cours du $\mathrm{XX}^{\mathrm{e}}$ siècle, l'industrialisation de la partie supérieure du bassin de la rivière Saguenay a conduit à une pollution significative de l'environnement marin en aval, particulièrement par les HAP provenant de l'industrie de l'aluminium (CRE, 1988). L'utilisation du procédé Söderberg dans les alumineries a fortement contribué à la contamination historique des 
sédiments du Saguenay par les HAP (LUN et al.,1998; MARTEL et al., 1987; SMITH et LEVY, 1990; WHITE et al., 1997). Au début des années 1980, les chercheurs de l'Université du Québec à Chicoutimi (MARTEL et al., 1986, 1987) ont mesuré dans les premiers centimètres de la couche sédimentaire des teneurs en HAP totaux variant de $16000 \mathrm{ng}_{\bullet} \mathrm{g}^{-1}$ dans le bras Nord à proximité de l'embouchure de la rivière Saguenay jusqu'à $2500 \mathrm{ng} \mathrm{g}^{-1}$ dans le bassin profond du fjord. Ces auteurs observèrent le début d'une décroissance des teneurs en HAP dans la couche superficielle qu'ils attribuèrent à une réduction récente des apports industriels. Des carottes de sédiments, prélevées par notre équipe dans le fjord du Saguenay en 1988, montrèrent des concentrations en HAP totaux variant de 2000 à $17000 \mathrm{ng}_{\bullet} \mathrm{g}^{-1}$ selon la profondeur et confirmèrent une réduction sensible du niveau de contamination dans la couche de surface (OUELLET, 1990). Cependant, la majorité des études postérieures à celle de MARTEL et collaborateurs mirent l'emphase sur le compartiment sédimentaire des contaminants sans porter attention ni à la matière particulaire en suspension (MPS) dans la colonne d'eau, ni aux HAP bioaccumulés dans le compartiment biotique. Seuls les travaux de WHITE et al. (1997) ont recherché les effets toxiques possibles des hydrocarbures sur la mye commune (Mya arenaria) dans le fjord du Saguenay. Les concentrations en HAP totaux oscillaient alors entre 35 et $40 \mathrm{ng}_{\bullet} \mathrm{g}^{-1}$ tissus, avec une forte

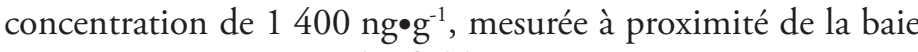
Sainte-Marguerite. Les plus faibles concentrations ( 4 à $5 \mathrm{ng}^{\circ} \mathrm{g}^{-1}$ ) ont été observées à l'embouchure du ford laissant supposer que les HAP étaient surtout retenus dans le fjord lui-même. Cependant, ces études ont porté seulement sur le fjord sans considérer la contribution de la MPS provenant de la rivière Saguenay, le long de laquelle sont situées les alumineries et les agglomérations urbaines.

Nous avons entrepris, au début des années 2000, une étude exhaustive pour caractériser en détail le profil chromatographique des HAP et les quantifier à la fois dans la rivière et le fjord du Saguenay avec une attention particulière à la contribution du bassin de drainage de la rivière en direction du fjord. Pour atteindre cet objectif, nous avons quantifié les HAP particulaires dans les eaux de la rivière pendant les saisons sans glace (8-9 mois). Les résultats ont ensuite été comparés avec ceux obtenus pour les eaux de surface et les sédiments superficiels du fjord afin d'établir un bilan de masse pour l'ensemble des apports annuels. Nous avons aussi examiné la distribution spatiale des HAP individuels dans les sédiments afin d'estimer les changements temporels des apports en HAP survenus au cours des 20 dernières années. Enfin, nous avons déterminé les HAP et certains de leurs métabolites dans quelques espèces d'invertébrés benthiques et épi-benthiques échantillonnés dans le ford afin d'estimer le niveau actuel de contamination de la faune benthique. Cet article constitue donc une synthèse des données acquises au cours de ces années et tente de faire le point sur l'évolution temporelle de la contamination par les HAP dans le système Saguenay, tout en comparant cette situation avec celle d'autres sites ailleurs dans le monde.

\section{MATÉRIELS ET MÉTHODES}

\subsection{Sites d'échantillonnage et conservation des échantillons}

Trois stations le long de la rivière Saguenay (Figure 1A) ont été échantillonnées afin de déterminer les teneurs en HAP dans la MPS des eaux douces de la rivière et en déduire l'influence possible des alumineries. La station A, localisée sur la partie fluviale de la rivière Saguenay, en amont du complexe industriel de la ville d'Alma, a été choisie afin d'obtenir le

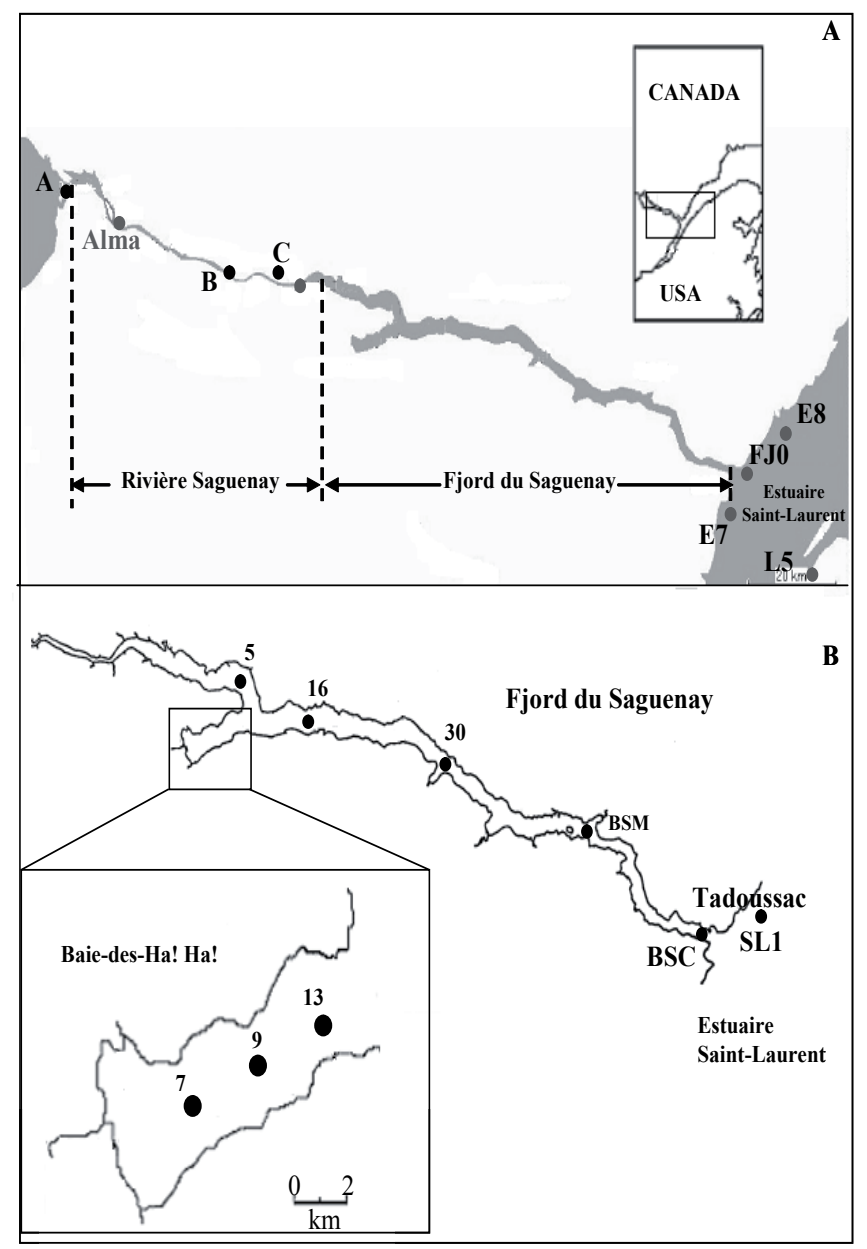

Figure 1. Carte du système Saguenay (A) à partir du lac Saint-Jean en amont jusqu'à l'estuaire du Saint-Laurent en aval, et du fjord du Saguenay (B) montrant les différentes stations d'échantillonnage. BSM : Baie-Sainte-Margerite; BSC : Baie-Sainte-Catherine.

Map of the Saguenay system (A) from upstream lake Saint-Jean to downstream St. Lawrence Estuary, and the Saguenay Fjord (B) showing different sampling stations: BSM: Baie Sainte-Margerite; BSC: Baie Sainte-Catherine. 
bruit de fond de la contamination sans influence directe des industries. La station B a été localisée au barrage Shipshaw d'Alcan Ltée, en aval de l'aluminerie d'Alma, mais en amont du complexe d'Arvida (ville de Saguenay) où des cuves de réduction Söderberg avec anodes pré-cuites verticales ont été en opération pendant plusieurs décennies jusqu'en 2005. La station C, localisée près de ville de Saguenay et proche de l'embouchure de la rivière sur le fjord, fut échantillonnée à marée basse seulement pour s'assurer d'obtenir de l'eau douce de la rivière seulement. Les trois sites ont été échantillonnés quatre fois en 2003 : les 11 et 12 mai (fin de l'hiver avant la crue du printemps); les 6 et 7 juin (fin du printemps); les 21 et 22 août (fin de la période tempérée de l'été) et le 25 novembre (fin de l'automne).

Pour le ford du Saguenay, des carottes de sédiments ont été obtenues en mai 2001 et 2002 au cours d'expéditions à bord du navire océanographique Alcide C. Horth. De même, les échantillons de MPS ont été obtenus en mai 2001 à partir d'échantillons d'eau prélevés avec des bouteilles Niskin immergées juste sous la surface. Les organismes benthiques et épi-benthiques, incluant des polychètes (Nereis sp.), des crevettes (Pandalus sp.), des myes (Mya arenaria) et des moules (Mytilus edulis) ont été échantillonnés avec un chalut à bâton au cours des expéditions de 2000, 2001 et 2002. Les sites d'échantillonnage (Figure 1B) pour le biote étaient localisés dans le bras Nord à proximité de l'embouchure de la rivière (station 5), considérée par le passé comme la principale source de HAP vers le fjord, dans le bassin supérieur du ford (stations 13 et 16) et dans le bassin inférieur proche du Saint-Laurent (station 30), la baie Sainte-Marguerite (BSM) et la baie Sainte-Catherine (BSC). Une autre station a aussi été échantillonnée dans la baie des Ha! Ha! (station 9), où la crue éclair de 1996 a apporté une épaisse couche de nouveaux sédiments d'origine terrigène recouvrant entièrement l'ancien lit sédimentaire (PELLETIER et al., 1999, 2003). Tous les échantillons biologiques furent entreposés à $-20^{\circ} \mathrm{C}$ dans des pots de verre pré-nettoyés à l'acétone/hexane jusqu'au moment de l'analyse.

Les carottes de sédiments ont été prélevées à l'aide d'un carottier multiple (Maxicorer Mark V-4000; Bowers and Connebly) à huit tubes (diamètre intérieur de $5 \mathrm{~cm}$ et longueur de $70 \mathrm{~cm}$ ). Immédiatement après l'échantillonnage, chaque carotte a été découpée en tranches de $0,5 \mathrm{~cm}$ pour les premiers $5 \mathrm{~cm}$ et en tranches de $3 \mathrm{~cm}$ pour le reste. Les échantillons ont été préservés dans des pots de verre et congelés à $-20^{\circ} \mathrm{C}$ sur le bateau.

Les échantillons d'eau de la rivière Saguenay obtenus en 2003 ont été filtrés sur des filtres Whatman GF/F $\mathrm{F}^{\odot}$ de porosité $0,7 \mu \mathrm{m}$ préalablement pesés et calcinés à $450^{\circ} \mathrm{C}$. Tous les filtres ont été gardés à $-20^{\circ} \mathrm{C}$ jusqu'au moment de la lyophilisation et ensuite conservés à $-80^{\circ} \mathrm{C}$. De même, l'eau de surface (0-1 m) du fjord du Saguenay a été échantillonnée en 2001 et filtrée sur les mêmes filtres Whatman GF/F $0,7 \mu \mathrm{m}$ et traitée de façon identique aux précédents. À cause de la faible quantité de MPS, les filtres provenant de la même station ont été groupés pour obtenir suffisamment de matériel pour l'analyse des HAP.

\subsection{Extraction et quantification des HAP particulaires}

Les HAP particulaires ont été extraits selon une méthode précédemment décrite par BRION et PELLETIER (2005). En résumé, $300 \mathrm{mg}$ de MPS ont été extraits avec $20 \mathrm{~mL}$ de dichlorométhane (DCM) au bain ultrasonique pendant une heure. Ensuite, les tubes ont été agités mécaniquement pendant deux heures pour finalement être soumis aux ultrasons pendant une autre période d'une heure. Après décantation du solvant, l'extraction aux ultrasons a été répétée avec un nouveau volume de $10 \mathrm{~mL}$ de DCM pour une heure. Les extraits d'un même échantillon ont été groupés, filtrés et évaporés sous un courant d'azote jusqu'à un volume de $0,5 \mathrm{~mL}$. Le concentré a été élué à travers une mini-colonne de nettoyage (Supelclean ENVI-18 SPE ${ }^{\text {тм }}$ de $3 \mathrm{~mL}$, SUPELCO) avec $5 \mathrm{~mL}$ du mélange 75:25 hexane:DCM. L'extrait a été de nouveau concentré sous courant d'azote jusqu'à $50 \mu \mathrm{L}$ en utilisant un bain de glace pour réduire la perte des HAP légers. Une solution d'étalons internes deutérés (naphtalène- $\mathrm{d}^{8}$, anthracène- $\mathrm{d}^{10}$ and pérylène- $\mathrm{d}^{12}$ ) a été ajoutée pour compléter le volume final à $100 \mu \mathrm{L}$.

La quantification des HAP a été obtenue par chromatographie gazeuse/spectrométrie de masse (ThermoFinnigan Polaris $\mathrm{Q} M S^{\circ}$ ) et la séparation a été assurée par une colonne capillaire Restek $^{\otimes}$ (30 m x 0,25 nm d.i.) avec l'hélium comme gaz porteur. La limite de détection était de $0,015 \mu \mathrm{g} \bullet \mathrm{mL}^{-1}$. Chaque HAP a été identifié et quantifié en utilisant son ion moléculaire et son temps de rétention par comparaison avec le mélange de standards HAP du ministère de l'Environnement du Québec (Supeco ${ }^{\circ}$ PAH Mixt 502065).

\subsection{Extraction et quantification des HAP des sédiments}

Les échantillons de sédiments obtenus en 2001 ( $1,0 \mathrm{~g}$ sec et en poudre) ont été extraits avec $10 \mathrm{~mL}$ de DCM au bain ultrasonique pendant $15 \mathrm{~min}$; une étape suivie par $18 \mathrm{~h}$ d'agitation mécanique et de nouveau 15 min aux ultrasons. Après centrifugation et décantation, les extraits ont été concentrés à $1 \mathrm{~mL}$ et élués sur mini-colonnes de nettoyage avec $10 \mathrm{~mL}$ du mélange hexane: DCM $(90: 10)$ pour éliminer la matière humique colorée. Les extraits ont été évaporés à sec sur un bain de glace et ensuite repris avec $1 \mathrm{~mL}$ d'acétonitrile. La quantification a été faite en chromatographie liquide avec détection en fluorescence en utilisant une colonne Supelcosil ${ }^{\mathrm{TM}}$ LC-PAH $(25 \mathrm{~cm} \times 3 \mathrm{~mm})$. Toutes les analyses ont été menées à débit constant $\left(0,8 \mathrm{~mL} \cdot \mathrm{min}^{-1}\right)$ avec un gradient d'eau nanopure et d'acétonitrile. Les longueurs d'ondes d'excitation 
et d'émission ont été fixées à $280 \mathrm{~nm}$ et $340 \mathrm{~nm}$ respectivement, pour les premières 5,5 min pour un maximum de sensibilité aux HAP légers. Ensuite, l'émission a été mesurée à $410 \mathrm{~nm}$ pour le reste du programme pour détecter les HAP plus lourds.

Pour les échantillons de sédiments obtenus en 2002, l'extraction a été faite sur $1,0 \mathrm{~g}$ de sédiments secs en poudre avec $10 \mathrm{~mL}$ de DCM par agitation mécanique pendant $16 \mathrm{~h}$, suivie par 30 min aux ultrasons. Le reste du protocole était identique à celui de la MPS avec l'utilisation de la chromatographie gazeuse couplée à la spectrométrie de masse.

\subsection{Détermination des HAP et métabolites dans les organismes biologiques}

Chaque échantillon de tissu biologique sec et homogénéisé (200 mg) a été extrait avec $5 \mathrm{~mL}$ du mélange hexane : acétone $(50: 50)$ au bain ultrasonique à $30^{\circ} \mathrm{C}$ pendant $30 \mathrm{~min}$, ensuite l'extrait a été agité mécaniquement pendant $3 \mathrm{~h}$ et enfin de nouveau soumis aux ultrasons pour 30 min. Après centrifugation et décantation, les extraits ont été nettoyés en utilisant un mélange hexane: acétone $(90: 10)$ sur minicolonne (Supelclean ENVI-18 ${ }^{\mathrm{TM}}$ SPE $3 \mathrm{~mL}$, SUPELCO) avec $1 \mathrm{~cm}$ de gel de silice ajouté en tête de colonne pour éliminer le maximum de matière organique colorée sans affecter l'élution des composés recherchés. L'extrait ainsi nettoyé a été réduit à $200 \mu \mathrm{L}$ sous un courant d'azote sur bain de glace.

Tous les HAP et les dérivés hydroxylés (HO-HAP) des tissus biologiques ont été analysés en utilisant le même extrait par chromatographie liquide/fluorescence. Pour les HO-HAP, la programmation de la pompe commençait à $65 \%$ d'acétonitrile (35\% eau nanopure) pendant 25 min pour ensuite augmenter jusqu'à $100 \%$ en $5 \mathrm{~min}$ avec un plateau final de $5 \mathrm{~min}$. Les longueurs d'ondes d'excitation et d'émission ont été fixées respectivement à $244 \mathrm{~nm}$ et $394 \mathrm{~nm}$. Deux composés hydroxylés typiques, le phénanthrénol et le pyrénol, ont été identifiés et quantifiés par l'utilisation de solutions étalons préparées à partir de composés purs achetés chez Sigma-Aldrich ${ }^{\oplus}$. La limite de détection était de $0,05 \mathrm{ng}_{\bullet} \mathrm{g}^{-1}$ p.s.

Pour l'ensemble des extractions et quantifications des HAP provenant de la MPS, des sédiments et des organismes, les contrôles de qualité ont été assurés par des injections successives de solutions standards de HAP permettant d'estimer une variabilité analytique égale ou inférieure à $\pm 12 \%$. La justesse des analyses a été vérifiée par l'analyse d'un standard de sédiment SRM $1941 \mathrm{~b}$ fourni par le National Institute of Standards and Technology (NIST).

Pour les tissus biologiques et les sédiments, la qualité des résultats a été vérifiée par l'addition interne de composés connus aux échantillons avant l'extraction et les rendements ont été calculés. Ceux-ci variaient entre 65 et $100 \%$ pour les tissus biologiques et de 75 à $105 \%$ pour les sédiments et la MPS. Les concentrations finales ont été corrigées en fonction des rendements obtenus pour chaque analyse. Tous les résultats sont exprimés en poids sec (p.s.) de l'échantillon.

\subsection{Analyses statistiques}

Les résultats des HAP individuels et des HAP totaux ont été comparés à l'aide d'une analyse ANOVA à un facteur afin de déterminer la variabilité de la distribution des HAP particulaires et pour détecter les différences entre les profils des HAP entre les stations et les saisons.

\section{RÉSULTATS ET DISCUSSION}

\subsection{Variation spatio-temporelle des HAP particulaires dans la rivière Saguenay}

La charge particulaire des eaux de la rivière Saguenay est généralement très faible avec des valeurs en MPS de l'ordre de 1,5 à $1,7 \mathrm{mg} \cdot \mathrm{L}^{-1}$ et varie peu selon le lieu d'échantillonnage et la saison. En utilisant un débit annuel moyen de $1369 \mathrm{~m}^{3} \cdot \mathrm{s}^{-1}$ (donnée de la société Alcan à la centrale Shipsaw), nous avons pu calculer que la charge annuelle de la MPS transportée par la rivière est d'environ 71450 tonnes, en supposant que la charge particulaire en hiver (sous le couvert de glace de décembre à mars inclusivement) n'est pas différente des autres saisons. La composition élémentaire de cette MPS varie au cours des saisons, le rapport $\mathrm{C} / \mathrm{N}$ étant de 8,4 à 9,3 en mai et juin et de 12,9 à 16,2 en novembre. Cette augmentation du $\mathrm{C} / \mathrm{N}$ en automne est conforme aux attentes et traduit la dégradation de la matière organique terrigène et lacustre avec la fin de la période de production végétale. La matière particulaire en suspension (MPS) provenant des échantillons d'eau douce de la rivière Saguenay contenait des HAP totaux variant de $3200 \mathrm{ng}_{\bullet} \mathrm{g}^{-1}$ en été (station B) à des valeurs atteignant

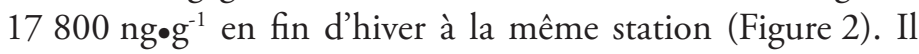
y a peu de différence entre les stations pour les saisons d'été et d'automne alors que les données d'hiver et de printemps apparaissent plus variables, notamment pour la station B qui est beaucoup plus faible au printemps que les autres mais nettement plus forte à la fin de l'hiver. L'abondance des HAP en fonction de la taille des molécules (nombre de cycles) suit généralement l'ordre : 2 cycles $>3$ cycles $>4$ cycles $>5$ cycles, à l'exception des échantillons d'automne qui montrent tous une domination des composés à 3 cycles (Figure 3). Nous n'avons pas observé un gradient longitudinal entre la station $\mathrm{A}$, située en amont des alumineries, et la station $\mathrm{C}$, où la contribution du complexe d'Arvida aurait pu être observable. La contamination 


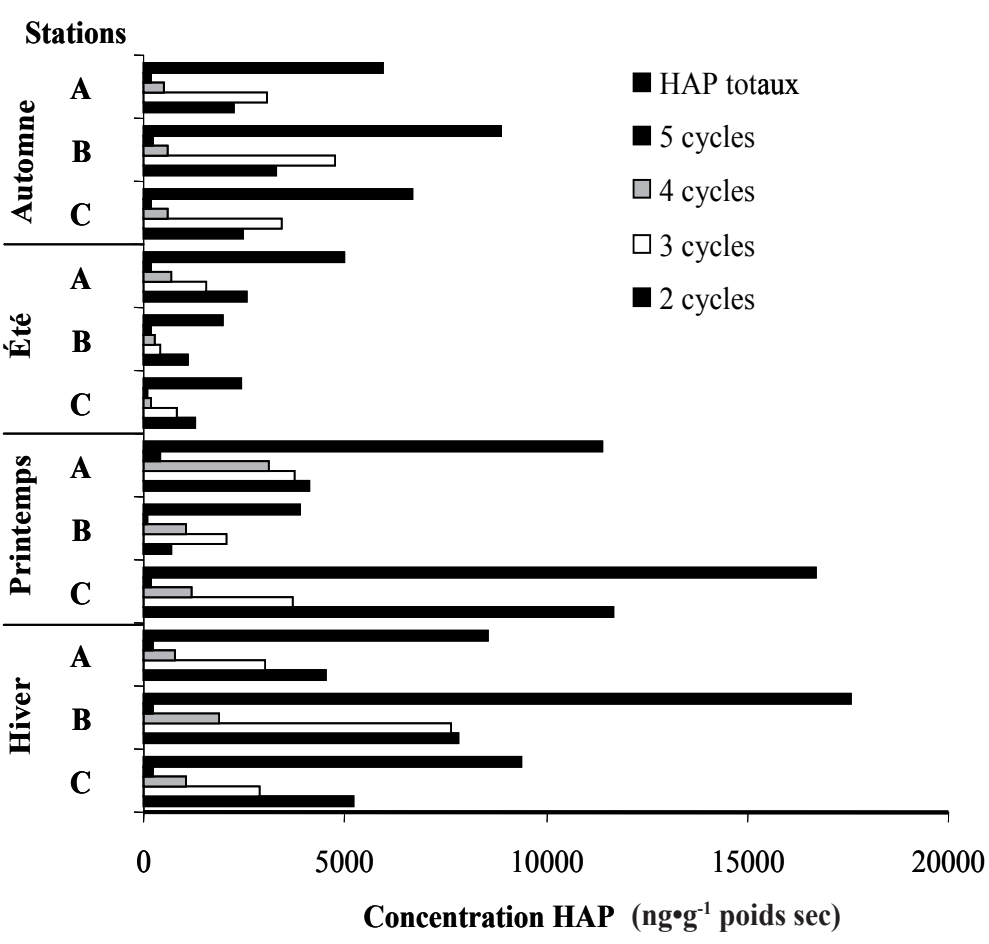

Figure 2. Distribution et des HAP totaux ( ng.g $^{-1}$ poids sec) et des HAP particulaires à 2, 3, 4 et 5 cycles dans les eaux de surface de la rivière Saguenay (Stations A, B et C) au cours de l'année 2003.

Distribution of total PAHs (ng. ${ }^{-1}$ dry weight) and particulate PAHs with 2, 3, 4 and 5 rings in Saguenay river surface waters (Stations A, B and C) during the year 2003.

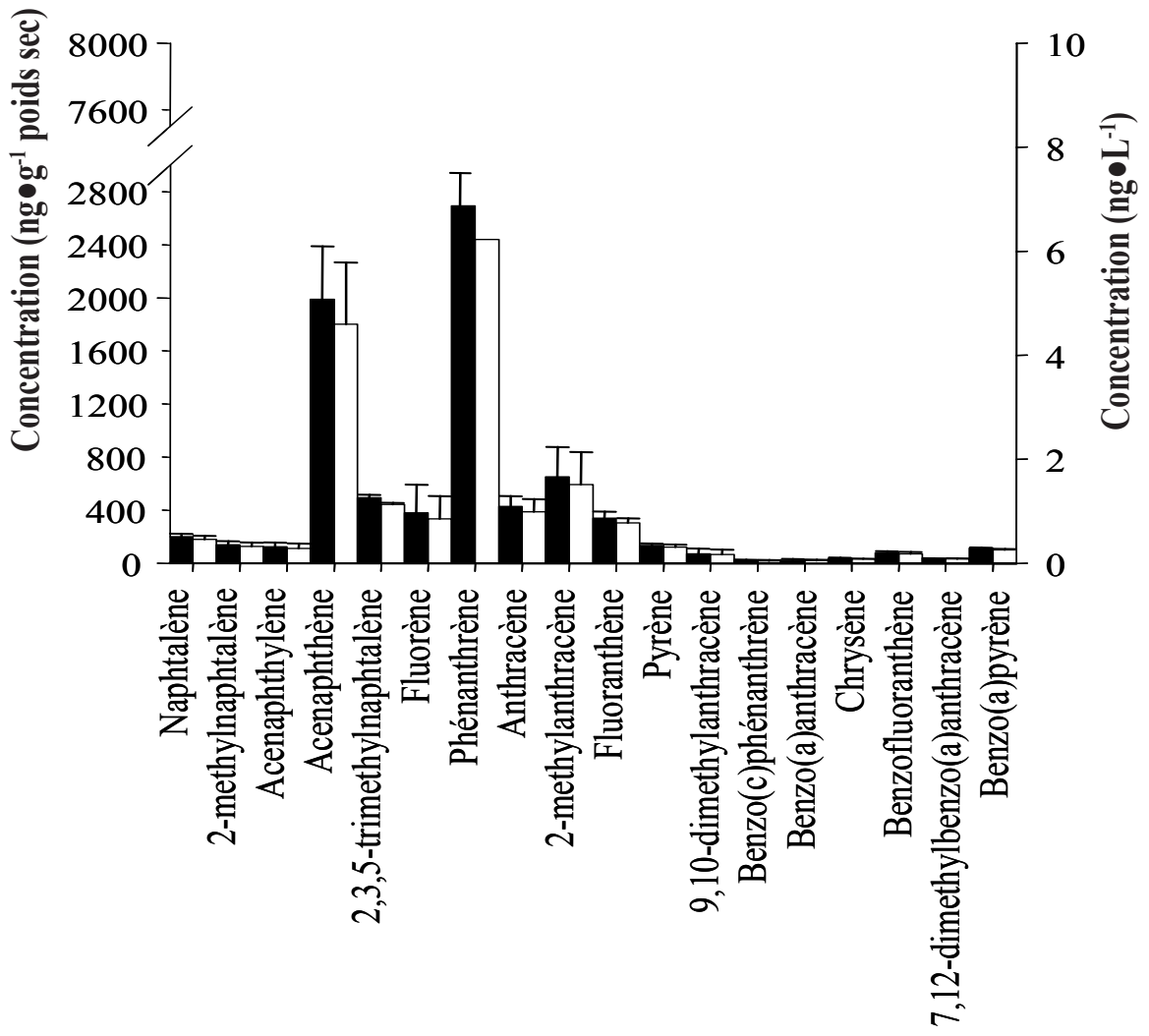

Figure 3. Exemple de la distribution des HAP particulaires échantillonnés à l'automne 2003. Les concentrations de HAP sont exprimées en fonction de la matière particulaire sèche ( ng. $^{-1}$ poids sec) (axe de gauche) et en fonction du volume d'eau (ng $\left.\mathrm{L}^{-1}\right)$ (axe de droite). (moyenne des 3 stations $\pm \mathrm{SD}$ ).

Example of the distribution of particulate PAHs sampled in Fall 2003. PAH concentrations are expressed as a function of particulate matter (ng॰g ${ }^{-1}$ dry weight) (left axis) and as a function of water volume (ng $L^{-1}$ ) (right axis). (Mean of all 3 stations $\pm S D)$. 
en HAP particulaires est plus élevée à la station $\mathrm{C}$ au printemps seulement.

En l'absence d'un gradient spatial le long de la rivière, nous avons groupé les données des trois stations et examiné leurs variations saisonnières. La figure 3 présente l'exemple de la distribution des HAP en automne à la fois en concentration massique (ng g $^{-1}$ poids sec) etenconcentrationvolumique $\left(n g \cdot \mathrm{L}^{-1}\right)$. La MPS échantillonnée à la fin de l'hiver et en automne montre des distributions assez similaires de HAP avec une domination de l'acénaphthène $\left(5\right.$ à $\left.6 \mathrm{ng} \cdot \mathrm{L}^{-1}\right)$ et du phénanthrène (4 à $7 \mathrm{ng} \cdot \mathrm{L}^{-1}$ ) alors que la MPS de l'été (non montrée) présente une distribution plus diversifiée des HAP mais ceux-ci sont beaucoup moins concentrés par rapport au volume d'eau (entre 0,1 et $0,4 \mathrm{ng} \cdot \mathrm{L}^{-1}$ ). La similitude entre le patron des HAP en automne et à la fin de l'hiver pourrait s'expliquer par la présence de neige et de glace dans les deux cas. Le couvert de neige retiendrait les apports atmosphériques récents et les analyses montreraient surtout le patron des HAP présents dans l'ensemble des particules provenant de la remise en suspension et de l'érosion du bassin versant.

L'examen des composés individuels montre que les HAP légers ( 2 et 3 cycles) sont dominants et représentent entre 80 et $90 \%$ des HAP totaux, quelle que soit la saison. Parmi eux, l'acénaphthène et le phénanthrène sont dominants (sauf pour l'été) avec des concentrations respectives variant de

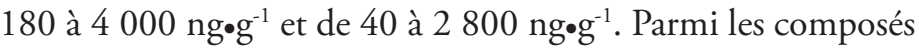
lourds ( 4 et 5 cycles), le benzo(a)pyrène $(\mathrm{BaP})$ apparaît souvent comme le plus abondant avec une concentration variant

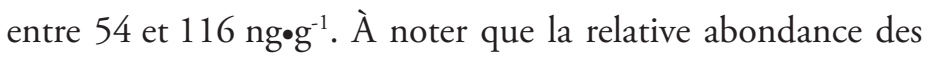
composés légers comme le naphtalène et le méthylnaphtalène dans la MPS de l'été pourrait indiquer une contamination d'origine pétrolière provenant, entre autres, de la navigation de plaisance. L'essence et le diesel contiennent surtout des alcanes aliphatiques et cycliques mais aussi une proportion de $10-20 \%$ de HAP légers (2-3 cycles). On y retrouve des naphtalènes et des phénanthrènes, ainsi que de l'acenaphthène et du fluoranthène qui s'échappent dans l'air (TAVARES et al., 2004) ou qui se dissolvent dans l'eau. Il est à noter que la solubilité du naphtalène dans l'eau est d'environ $30 \mathrm{mg} \cdot \mathrm{L}^{-1}$ alors que celle de l'acénaphthène est de l'ordre de $4-5 \mathrm{mg} \cdot \mathrm{L}^{-1}$. Le phénanthrène est soluble au niveau de 1 à $1,5 \mathrm{mg} \mathrm{L}^{-1}$ (MACKAY et al., 1992).

Les HAP dissous dans l'eau ou dispersés par une action mécanique, comme l'hélice d'un moteur hors bord, auront tendance à se réadsorber sur les particules en suspension selon la nature même de ces particules et les conditions physicochimiques du milieu (température, force ionique, matière organique dissoute, etc.). Il n'est donc pas étonnant de retrouver des hydrocarbures pétrogéniques sur le matériel particulaire en suspension de la rivière Saguenay. Il est cependant impossible de discriminer entre les HAP légers provenant des carburants pétroliers et les HAP légers provenant d'un procédé industriel quelconque comme une aluminerie ou même de la combustion de végétaux pendant un incendie de forêts.

Nos données pour la rivière Saguenay ont été comparées à celles de quelques rivières et estuaires dans le monde

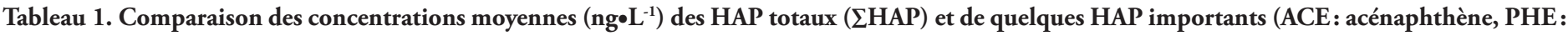
phénanthrène, CHR: chrysène) dans la matière particulaire en suspension entre la rivière Saguenay et quelques autres rivières et estuaires dans le monde. Le rapport phénanthrène et les HAP totaux (PHE/ $\Sigma$ HAP) est aussi donné.

Table 1. Comparison of mean concentrations (ng $\left.L^{-1}\right)$ of total PAHs ( $\mathrm{HAP}$ ) and some important PAHs (ACE: acenaphthene, PHE: phenanthrene, CHR: chrysene) in suspended particulate matter of the Saguenay River and a number of rivers and estuaries around the world. The ratio between phenanthrene and total PAHs ((PHE/乏HAP) is also reported.

\begin{tabular}{|c|c|c|c|c|c|c|c|}
\hline Emplacement & $\begin{array}{c}\text { Période } \\
\text { d'échantillonnage }\end{array}$ & $\begin{array}{c}\text { Principaux HAP } \\
\text { observés }\end{array}$ & ACE & PHE & $\mathrm{CHR}$ & $\Sigma \mathrm{HAP}$ & $\mathrm{PHE} / \Sigma \mathrm{HAP}$ \\
\hline Estuaire Jiulong (Mer Xiamen, Chine) ${ }^{1}$ & Juin 1999 & 5 cycles & $110-5700$ & $160-1370$ & 1070 & $6960-26900$ & $0,01-0,08$ \\
\hline Baie Izmit (Turquie) ${ }^{2}$ & Août 1999 & 4 cycles & 0,29 & $0,19-2,47$ & $0,29-1,17$ & $0,16-7,40$ & $0,06-1,0$ \\
\hline Rivière Thermaikos (Grèce) ${ }^{3}$ & Juillet-août 1996 & 2 cycles & n.d. & $30-70$ & $0,9-45$ & $184-856$ & $0,06-0,17$ \\
\hline Rivière Elizabeth (États-Unis) ${ }^{4}$ & Octobre 1993 & 4-5 cycles & $0,16-0,54$ & $0,88-2,31$ & $0,90-3,95$ & 25,4 & n.d. \\
\hline Rivière York (Etats-Unis) ${ }^{4}$ & Octobre 1993 & $4-5$ cycles & $0,04-0,14$ & $0,24-0,78$ & $0,16-0,78$ & 6,24 & n.d. \\
\hline Rivière Seine (France) $)^{5}$ & Octobre 1993 & 5 cycles & n.d. & $0,6-82$ & n.d. & $2-687$ & $0,03-0,12$ \\
\hline Rivière Saguenay (Canada) ${ }^{6}$ & Juin 2003 & $2-3$ cycles & $0,33-8,33$ & $0,32-1,29$ & 0,03-0,09 & $6,23-17,5$ & $0,02-0,21$ \\
\hline Rivière Saguenay (Canada) ${ }^{6}$ & Août 2003 & $2-3$ cycles & $0,09-0,10$ & $0,08-0,09$ & 0,01 & $1,35-2,19$ & $0,01-0,02$ \\
\hline
\end{tabular}

${ }^{1}$ MASKAOUI et al. (2002); ${ }^{2}$ TELLI-KARAKOÇ et al. (2002); ${ }^{3}$ MANOLI et al. (2000); ${ }^{4}$ GUSTAFSON et GSCHWEND (1997); ${ }^{5}$ FERNANDES et al. (1997), ${ }^{6}$ Notre étude.

n.d. = non disponible 


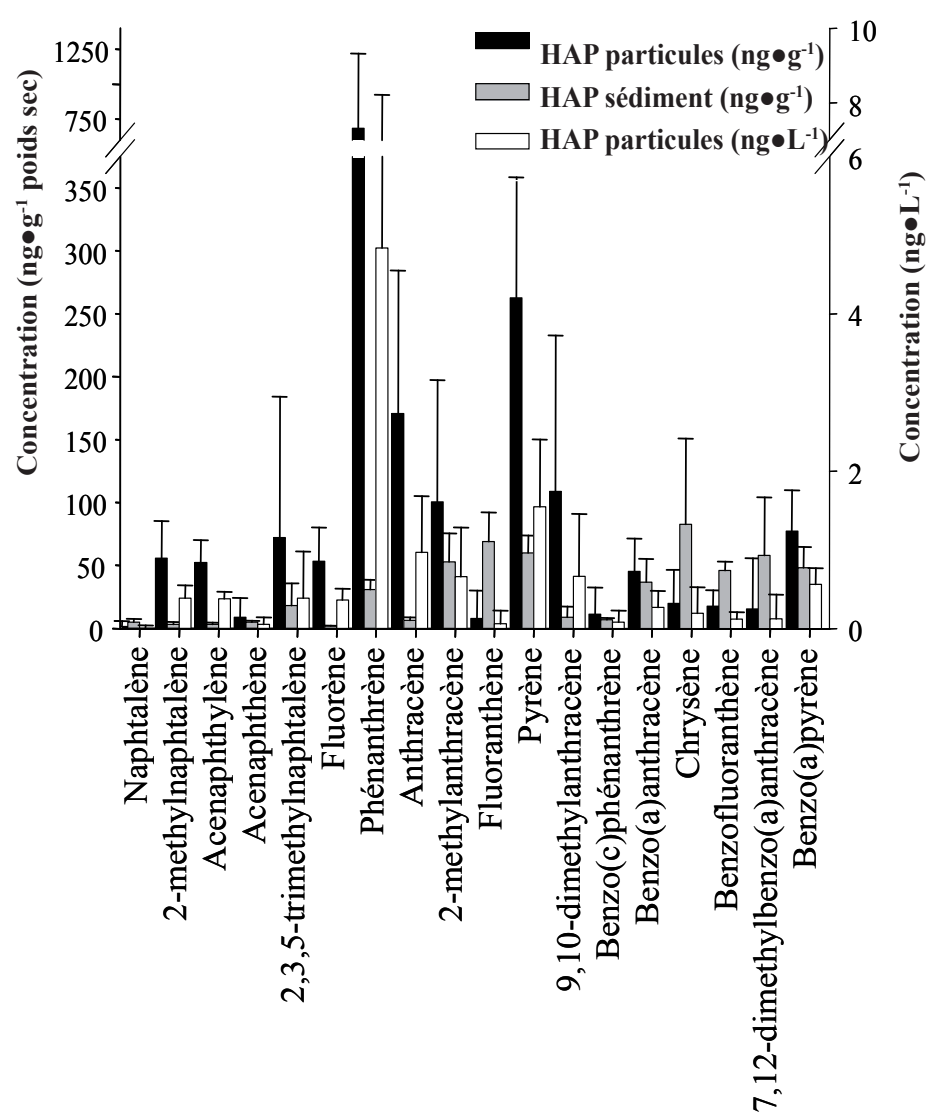

Figure 4. Distribution des HAP particulaires dans les eaux de surface (données en ng॰g $^{-1}$ poids sec et ng• $\mathrm{L}^{-1}$ ) et les sédiments de surface (ng•g-1 poids sec) du fjord du Saguenay échantillonnés en mai 2001. (moyenne de toutes les stations \pm DS). Distribution of particulate PAHs in surface waters (data in $n g \bullet g^{-1}$ dry weight and $n g \bullet L^{-1}$ ) and in surface sediment (ng• $g^{-1}$ dry weight) of Saguenay Fjord sampled in May 2001 (Mean of all 3 stations \pm SD).

(Tableau 1). Une telle comparaison doit être faite avec prudence car les techniques d'échantillonnage et d'analyse different selon les auteurs et les données rapportées en HAP totaux ne correspondent pas toujours à la somme des mêmes HAP individuels. Les principaux HAP mesurés dans plusieurs des rivières du monde sont des composés lourds de 4 et 5 cycles comme le chrysène et le benzo(a)pyrène $(\mathrm{BaP})$ et diffèrent ainsi des composés légers à 2 ou 3 cycles généralement retrouvés dans la MPS de la rivière Saguenay. La gamme des HAP totaux observés dans la rivière Saguenay est nettement inférieure aux concentrations rapportées pour certains sites de Chine et de Grèce mais se retrouve assez similaire aux rivières américaines de l'Est et de la Turquie (Tableau 1). Le rapport entre le phénanthrène, un composé majeur observé fréquemment dans le Saguenay, et la somme des HAP (PHE/ $/ \mathrm{HAP}$ ) ne révèle pas un patron particulier pour la rivière Saguenay avec des valeurs variant entre 0,01 et 0,21 suivant les saisons, ce qui correspond aux rapports calculés pour les autres rivières et estuaires (Tableau 1). Ce rapport très variable est une autre indication des sources multiples d'hydrocarbures aromatiques dans la rivière Saguenay.

\subsection{Distribution des HAP particulaires dans le ford du Saguenay et comparaison avec la rivière}

Tout comme nous l'avons déjà observé pour la rivière, il n'y a pas de différence significative dans la concentration et la distribution des HAP particulaires mesurés dans les eaux de surface des différentes stations du fjord du Saguenay en 2001. La moyenne des concentrations a alors été faite et de nouveau exprimée à la fois en $n g \bullet \mathrm{g}^{-1}$ de MPS et en $n g \cdot \mathrm{L}^{-1}$ d'eau échantillonné (Figure 4). La valeur moyenne des HAP particulaires totaux était de $1764 \mathrm{ng} \mathrm{g}^{-1}$, soit des HAP de 2 à 10 fois moins concentrés que les observations faites dans la rivière pour toutes les saisons. La comparaison du patron de distribution des HAP particulaires de la surface du fjord (Figure 4) avec celui des HAP de la rivière (Figure 3) montre un changement radical entre les eaux douces et les eaux marines avec une perte des HAP légers par un ordre de grandeur et un gain relatif des HAP plus lourds comme le pyrène, le chrysène et le $\mathrm{BaP}$. Cette différence s'explique par divers facteurs physicochimiques. D'une part, l'augmentation de la salinité provoque 
un relarguage de certains composés organiques, réduit encore plus la solubilité des HAP et favorise donc leur adsorption sur les particules en suspension. Ainsi, des composés comme le phénanthrène et le pyrène, tous les deux relativement solubles en eau douce, auront tendance à fuir la phase aqueuse au moment de la dilution avec l'eau salée et s'adsorber sur les particules en suspension dans le ford. L'adsorption des HAP les plus lourds sur les particules est un mécanisme par étapes qui conduit à la séquestration dans les particules en suspension et à leur sédimentation. Nos travaux récents sur l'adsorption et la séquestration des HAP sur les sédiments du Saguenay nous ont permis de montrer que les HAP de trois et quatre cycles ont été les plus rapides à se séquestrer et devenir peu accessibles aux organismes (BRION et PELLETIER, 2005). D'autre part, l'activité microbienne contribue fortement à la diminution des HAP légers dans les sédiments puisque les HAP ayant un petit nombre de cycles aromatiques sont plus facilement dégradés par les bactéries dans la colonne d'eau, ce qui réduit le nombre de ces molécules à atteindre le fond (LEI et al., 2005). Enfin, notons que les HAP légers étant plus solubles, ils auront tendance à l'évaporation et à la dilution dans la masse d'eau (KHIM et al., 2001).

La dernière étape de ce travail sur la MPS a consisté à calculer la contribution globale en HAP de la rivière en utilisant une approche de bilan de masse. Les données sur la charge particulaire transportée par la rivière pour les saisons échantillonnées ne sont pas disponibles, cependant, les auteurs FORTIN et PELLETIER (1995) ont calculé un transport annuel en MPS de 160000 tonnes pour la rivière Saguenay. Cette valeur differe sensiblement de la valeur de 71450 tonnes calculée précédemment à partir de nos propres valeurs de MPS. En faisant la moyenne générale de toutes nos données de HAP particulaires pour toutes les saisons échantillonnées en 2003 dans la rivière, nous obtenons une valeur moyenne

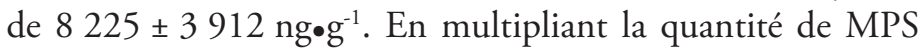
transportée annuellement par la rivière selon FORTIN et PELLETIER (1995), par la charge particulaire en HAP, on obtient une contribution estimée à $1300 \pm 625 \mathrm{~kg} \mathrm{an}^{-1}$. Le phénanthrène et le $\mathrm{BaP}$ sont quant à eux estimés à $255 \pm 120$ et $14 \pm 6 \mathrm{~kg} \bullet \mathrm{an}^{-1}$, respectivement. Par contre, si on utilise notre valeur annuelle de 75450 tonnes de MPS, on obtient

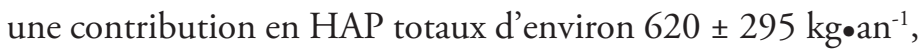
soit une valeur d'un peu plus d'une demi-tonne annuellement. En fait, les deux estimations ne sont pas significativement différentes à cause de la forte variabilité de la concentration des HAP totaux selon les échantillonnages successifs. Une valeur approximative d'une tonne de HAP apportée annuellement par la rivière pourrait constituer une estimation raisonnable. Pour fin de comparaison, notons que la baie de San Francisco reçoit annuellement une charge en HAP totaux pouvant atteindre 10,5 tonnes (OROS et al., 2007).
Il n'existe aucune donnée antérieure sur le transport des HAP particulaires par la rivière Saguenay. À partir des données de production d'aluminium, on estime qu'au moins 100 tonnes de HAP étaient déversées chaque année dans le système hydrographique du Saguenay avant la mise en place de mesures de rétention des suies et boues d'épurateurs en 1976 (MARTEL et al., 1986; SMITH et LEVY, 1990). Même si tous ces HAP n'étaient pas transportés sous une forme particulaire par la rivière Saguenay vers le ford, il est certain que la contribution actuellement estimée à une tonne par année est très faible par rapport à la période de forte contamination des années 1950 à 1970 .

\subsection{Distribution des HAP dans la phase sédimentaire}

La concentration des HAP individuels trouvés dans les sédiments de surface du ford est moindre que celle de la MPS (Figure 4). La concentration moyenne des HAP totaux (toutes

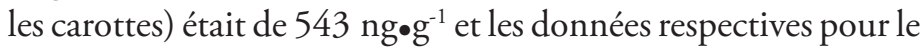
phénanthrène et le $\mathrm{BaP}$ étaient de 31 et 48 ng g $^{-1}$. On remarque immédiatement que la distribution des HAP dans les sédiments differe beaucoup de celle de la MPS (HAP particules) avec une plus forte contribution des composés à 4 et 5 cycles (Figure 4). On voit surtout la perte du phénanthrène, de l'anthracène et du pyrène et le gain en chrysène, benzoanthracène et méthylbenz $o$ (a)anthracène. On remarque aussi une quasi-conservation du benzo(a)pyrène à travers le système puisqu'il était déjà présent dans les particules transportées par la rivière à une valeur de $85 \mathrm{ng} \mathrm{g}^{-1}$ (Figure 3 à droite) et qu'on en retrouve entre 23 et $74 \mathrm{ng} \bullet \mathrm{g}^{-1}$ dans le premier $\mathrm{cm}$ des sédiments du fjord. Une telle importance des composés ayant de hauts poids moléculaires est commune à la majorité des sédiments lacustres et marins provenant de secteurs influencés par l'activité humaine (BATES et al., 1987; PEREIRA et al., 1996; READMAN et al., 1987). Le caractère hautement hydrophobe de ces composés accroît leurs interactions avec les particules en suspension et conduit à leur persistance dans la phase sédimentaire (ZHOU et ROWLAND, 1997).

Nos résultats illustrent l'un des rares exemples du processus biogéochimique que subissent les HAP lors de la transition entre les eaux douces d'une rivière et le mélange avec les eaux estuariennes. Alors que la rivière Saguenay apporte surtout de l'acénaphthène, du phénanthrène et quelques autres composés à 2 et 3 cycles à des concentrations parfois au-dessus de $2 \mu \mathrm{g} \bullet \mathrm{g}^{-1}$, les sédiments de surface du fjord conservent principalement les composés à 4 et 5 cycles dont le benzo(a)pyrène. La cancérogénicité des HAP lourds est bien documentée chez les mammiferes de laboratoire et chez les humains (STRAIF et al., 2005) mais il y a peu de liens directs qui puissent être établis entre la présence des HAP dans un environnement aquatique donné et le développement du cancer chez les organismes 
qui habitent cet environnement. De multiples travaux ont cependant montrél'induction des enzymes de type cytochromes P450 et la formation d'adduits des HAP hydroxylés avec l'ADN et autres protéines d'importance (VAN DER OOST et al., 2003). Dans une étude menée sur les causes de mortalité des bélugas (Delphinapterus leuca) du Saint-Laurent, les auteurs ont attribué à la présence historique des HAP dans les sédiments du Saguenay le développement de néoplasies gastrointestinales chez quelques-uns de ces animaux (MARTINEAU et al., 2002). Des travaux sur le transfert trophique des HAP et de leurs métabolites ont été entrepris au cours des dernières années afin de vérifier l'hypothèse selon laquelle la nourriture pourrait constituer une source de HAP pour une proie dans un environnement contaminé (COUILLARD, 2008; COUILLARD et al., 2009).

\subsection{Evolution temporelle des HAP dans les sédiments du ford}

En utilisant l'échantillonnage des carottes de sédiments de 2002, il nous a été possible de comparer le niveau de la contamination du début du $\mathrm{XXI}^{\mathrm{e}}$ siècle avec les données publiées par MARTEL et al. $(1986,1987)$ qui correspondaient à des carottes prélevées en 1982. Les HAP sont encore présents à toutes les profondeurs des quatre carottes étudiées (Figure
5). Les concentrations varient grandement entre les carottes en fonction de leur localisation dans le fjord. Pour la station 5, échantillonnée dans le bras Nord du fjord à quelques $\mathrm{km}$ de ville de Saguenay (anciennement Chicoutimi), on observe des concentrations entre 450 et $600 \mathrm{ng} \mathrm{g}^{-1}$ sur une profondeur d'environ $20 \mathrm{~cm}$ juste avant une augmentation brusque à plus

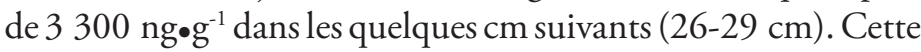
station a une profondeur d'eau d'environ $100 \mathrm{~m}$ et possède un fort taux de sédimentation d'environ $2,2 \mathrm{~cm} \cdot \mathrm{an}^{-1}$ selon les données de LECLERC et al. (1986). Assumant que le taux de sédimentation dans le ford n'a pas changé significativement depuis les années 1980 (aucune modification majeure du réseau hydrogéologique n'est connue) et que la crue éclair de 1996 n'a apporté qu'une contribution mineure de sédiments au bras Nord (2-3 cm selon des estimations visuelles obtenues en 1997 , résultats non publiés), on peut raisonnablement supposer que les premiers $22 \mathrm{~cm}$ de la carotte 5 représentent la contribution sédimentaire de la rivière Saguenay au cours des derniers 10 à 15 ans, compte tenu de la compression naturelle de la couche sédimentaire. Ceci met en évidence une contribution relativement faible et constante en HAP particulaires provenant de la rivière depuis le milieu des années 1980 . Ce résultat est en accord avec les conclusions de MARTEL et al. $(1986,1987)$ qui remarquaient déjà dans les carottes de 1982 une réduction tangible des HAP provenant des alumineries. La réduction

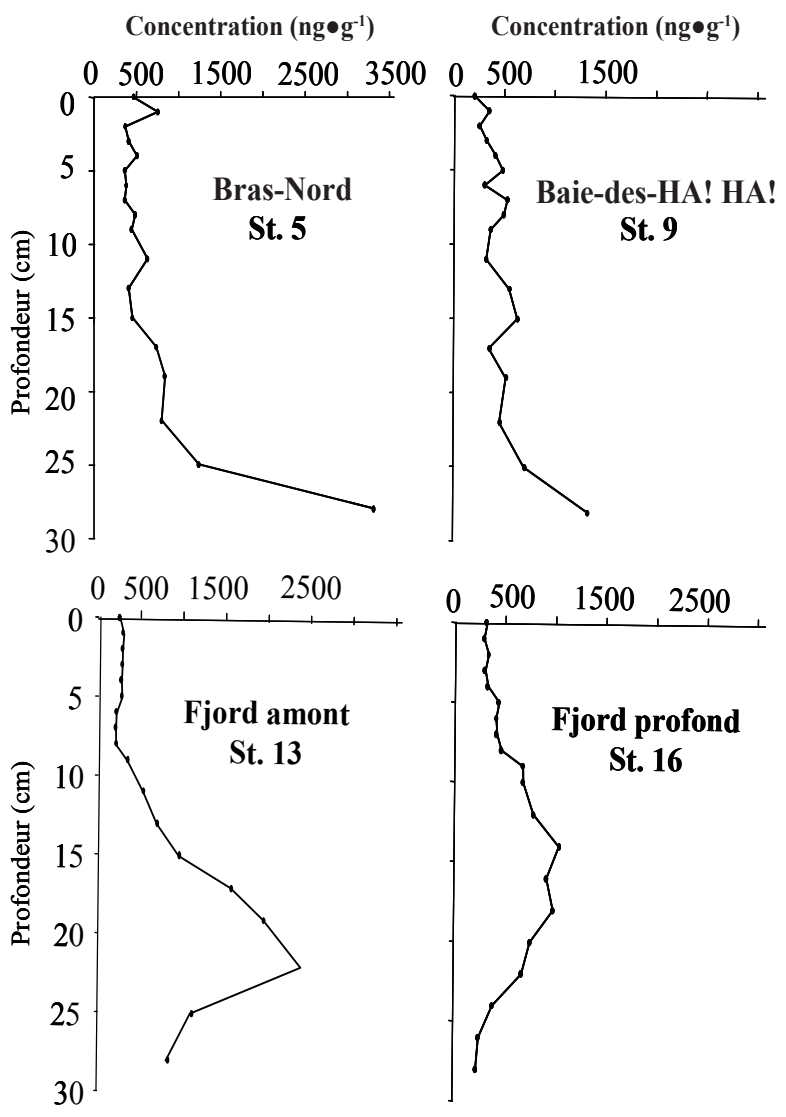

Figure 5. Quatre profils de HAP totaux en fonction de la profondeur (cm) dans des carottes échantillonnées en 2001 et 2002 dans le fjord du Saguenay.

Four profiles of total PAHs as a function of depth (cm) in cores collected in 2001 and 2002 in the Saguenay Fjord. 
Tableau 2. Comparaison des concentrations des HAP totaux (ng•g-1 poids sec) déterminées dans les premiers $2-3 \mathrm{~cm}$ des sédiments du fjord du Saguenay échantillonnés en mai 2002 avec les données publiées par MARTEL et al. (1987) pour des échantillons pris en avril 1982 à des sites très proches de nos propres stations.

Table 2. Comparison of total PAH concentrations (ng $\mathrm{g}^{-1}$ dry weight) determined in the first 2-3 cm of the Saguenay Fjord sediments sampled in May 2002 with results published by MARTEL et al. (1987) for samples taken in April 1982 at locations very close to our own stations.

\begin{tabular}{lccccc}
\hline Sources des données & $\begin{array}{c}\text { St. 5 } \\
(\text { St G })^{1}\end{array}$ & $\begin{array}{c}\text { St. } 9 \\
(\text { St H })\end{array}$ & $\begin{array}{c}\text { St. 13 } \\
(\text { St E })\end{array}$ & $\begin{array}{c}\text { St. 16 } \\
(\text { St D })\end{array}$ & $\begin{array}{c}\text { St. 30 } \\
(\text { St B })\end{array}$ \\
\hline Présente étude & 496 & 273 & 266 & 308 & 329 \\
MARTEL et al. (1987) & 4000 & 3500 & 7000 & 5000 & 3500 \\
\hline Facteur de décroissance & 8,1 & 12,8 & 26,3 & 16,2 & 10,6 \\
\hline
\end{tabular}

${ }^{1}$ Identification des sites correspondants dans l'étude de MARTEL et al. (1987)

des rejets hydriques a été entreprise au début des années 1970 avec la construction de bassins de rétention et de décantation des eaux usées des usines d'Arvida et surtout d'Île-Maligne. À la même époque, des modifications ont été apportées au système de ventilation des salles de cuves afin de réduire le rejet dans l'atmosphère des fines particules de suie provenant des opérations d'électrolyse. Une autre carotte prélevée en 1979 dans le même secteur que notre carotte 5, mais dont les résultats ont été publiés en 1990 seulement (SMITH et LEVY, 1990), a permis de calculer que le flux des HAP totaux vers les sédiments du ford a diminué d'environ $130 \mu \mathrm{g} \bullet \mathrm{cm}^{-2} \bullet \mathrm{an}^{-1}$ en 1974 à moins de $45 \mu \mathrm{g} \mathrm{cm}^{-2} \mathrm{an}^{-1}$ en 1979. Les mêmes auteurs avaient observé une pointe de plus $200000{\mathrm{ng} \bullet \mathrm{g}^{-1}}^{-1}$ vers 1970 alors que MARTEL et al. (1986) avaient trouvé un

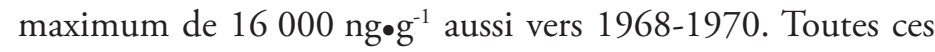
données montrent l'ampleur des rejets industriels de HAP dans la rivière Saguenay dans les années 1960 et 1970 ainsi que la réduction très marquée de ces apports dans les années 1980 .

À mesure qu'on s'éloigne de l'embouchure de la rivière (stations 16 et 30), le pic maximum se rapproche de la surface et s'atténue à cause d'un taux de sédimentation toujours plus faible et d'une certaine homogénéisation causée par la bioturbation, un processus biogéochimique bien connu depuis longtemps (KRANTZBERG, 1985) et capable de transporter verticalement les composés organiques hydrophobes comme les HAP (THIBODEAUX et al., 2001). La station 13 (Figure 5) montre un profil très net de l'évolution récente des HAP transportés dans le Saguenay au cours des six dernières décennies. Les concentrations montrent un plateau en dessous de $400 \mathrm{ng} \mathrm{g}^{-1}$ pour les premiers $8-10 \mathrm{~cm}$, suivi d'une croissance régulière jusqu'à $2400 \mathrm{ng}_{\bullet} \mathrm{g}^{-1}$ à $22 \mathrm{~cm}$ et d'une décroissance finale à $820 \mathrm{ng} \mathrm{g}^{-1}$ à $28 \mathrm{~cm}$. Le taux de sédimentation dans ce secteur est estimé à $0,5 \mathrm{~cm} \cdot \mathrm{an}^{-1}$ (LECLERC et al., 1986). Ce profil est une indication claire de l'état stable des faibles apports des HAP au ford au cours des 20 dernières années, les apports maximums ayant été atteints au cours des années 1960 comme déjà démontré par MARTEL et al. (1987). La station 16 présente

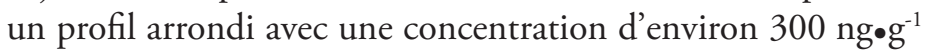
en surface, suivie d'un pic évasé vers $14-17 \mathrm{~cm}$, correspondant aux apports massifs des années 1960. Dans ce secteur profond du fjord, les taux de sédimentation sont de 0,2 à $0,3 \mathrm{~cm} \cdot \mathrm{an}^{-1}$ (MARTEL et al., 1987). Les faibles taux de sédimentation combinés à l'activité des organismes benthiques ont contribué à aplanir le pic des sédiments contaminés. Il en résulte une concentration maximale un peu inférieure à 1000 ng• $\mathrm{g}^{-1}$ à une profondeur de $14 \mathrm{~cm}$. Pour fin de comparaison, notons que MARTEL et al. (1987) avaient mesuré une concentration de $7000 \mathrm{ng} \cdot \mathrm{g}^{-1}$ pour une carotte prélevée au même endroit en 1982. La dilution par bioturbation et la biodégradation ont réduit de sept fois la concentration maximale observée dans ce bassin profond du ford.

La baie des Ha! Ha! présente un taux de sédimentation faible de l'ordre de $0,15 \mathrm{~cm} \bullet \mathrm{an}^{-1}$ (LECLERC et al., 1986) parce qu'elle n'a pas d'affluents importants et qu'elle se trouve en retrait des apports massifs de la rivière Saguenay. En 1982, MARTEL et al. (1987) ont observé un important pic de HAP tout proche de la surface et qui atteignait $3800 \mathrm{ng} \bullet \mathrm{g}^{-1}$. Ce pic témoignait du très faible taux d'enfouissement des HAP qui avaient été déposés dans cette baie au cours des décennies précédentes. Vingt ans plus tard, on pouvait s'attendre à une présence encore importante des HAP à peu de profondeur (5-6 cm) mais on y retrouve en fait des sédiments très faiblement contaminés $\left(<500 \mathrm{ng} \bullet \mathrm{g}^{-1}\right)$ sur toute la carotte jusqu’à $25 \mathrm{~cm}$ (Figure 5). Ce profil a été clairement influencé par l'apport d'une couche sédimentaire de 10-15 cm transportée par la crue éclair de 1996 qui a particulièrement touché la baie des $\mathrm{Ha}$ ! Ha! (PELLETIER et al., 1999; VIGLINO et al., 2004). Le pic de HAP a été enfoui sous cette couche de sédiments propres et semble y être stable à long terme (PELLETIER et al., 2003)

En résumé, on constate que les concentrations de HAP dans les sédiments de surface en 2002 sont de 10 à 30 fois plus 
faibles que celles publiées par MARTEL et al. (1987) pour des échantillons de 1982 (Tableau 2). En assumant que les taux de sédimentation n'ont pas changé, on peut estimer que les apports actuels en HAP aux sédiments de surface du fjord sont aussi 10 à 30 fois inférieurs à ceux calculés par MARTEL et al. (1987) pour 1980, soit de l'ordre de $60 \pm 30 \mathrm{~kg} \bullet \mathrm{an}^{-1}$ pour l'ensemble du fjord. Cette valeur est très inférieure (moins de $1 \%$ ) aux apports que nous avons estimés (environ 1 t•an $^{-1}$ ) à partir de la MPS de la rivière. Les raisons en sont essentiellement de trois ordres : 1) la perte des composés légers (deux et trois cycles surtout) par évaporation et dissolution; 2) l'advection d'une portion de la MPS hors du Saguenay par le mouvement des courants et des marées; 3) la biodégradation lente des HAP par la flore bactérienne et la faune pélagique et benthique. Avec les données disponibles, il est impossible d'établir l'importance relative de chacun des puits de HAP dans le Saguenay.

La source principale des HAP présents dans des sédiments lacustres ou marins peut être évaluée à l'aide des rapports entre les concentrations de certains HAP individuels comme le phénanthrène/anthracène (PHE/ANT). Les processus de combustion (feux de forêts et procédés industriels) donnent des valeurs de PHE/ANT $<10$ alors que les processus de maturation lente comme la diagénèse du pétrole donnent des
PHE/ANT > 15. Le rapport fluoranthène/pyrène (FLU/PYR) fournit une information similaire sur la source possible des HAP. Un ratio FLU/PYR de 0,8 à 1 est généré par un processus pyrolytique alors qu'un rapport $<0,8$ est l'indication d'une source pétrogénique (BAUMARD et al., 1998). En considérant toutes les analyses de sédiments que nous avons effectuées dans les carottes de 2002, on obtient une valeur moyenne de 4,64 $( \pm 1,48)$ pour le ratio PHE/ANT et $1,11( \pm 0,18)$ pour le ratio FLU/PYR. La figure 6 montre la distribution des valeurs individuelles de ces deux rapports. Ces données confirment l'origine pyronégique pour les HAP de la couche sédimentaire du Saguenay, même pour la couche de surface actuelle. En fait, les apports plutôt pétrogéniques que nous avons décrits dans la rivière Saguenay n'atteignent pas vraiment le fond du fjord. La contamination probable des eaux du Saguenay par les hydrocarbures pétroliers ne se répercute pas clairement sur la composition des sédiments profonds.

\subsection{Distribution des HAP dans le biote}

Afin de tenter d'évaluer les interactions possibles entre les HAP analysés dans la MPS et les sédiments et quelques organismes benthiques, nous avons analysé les HAP individuels

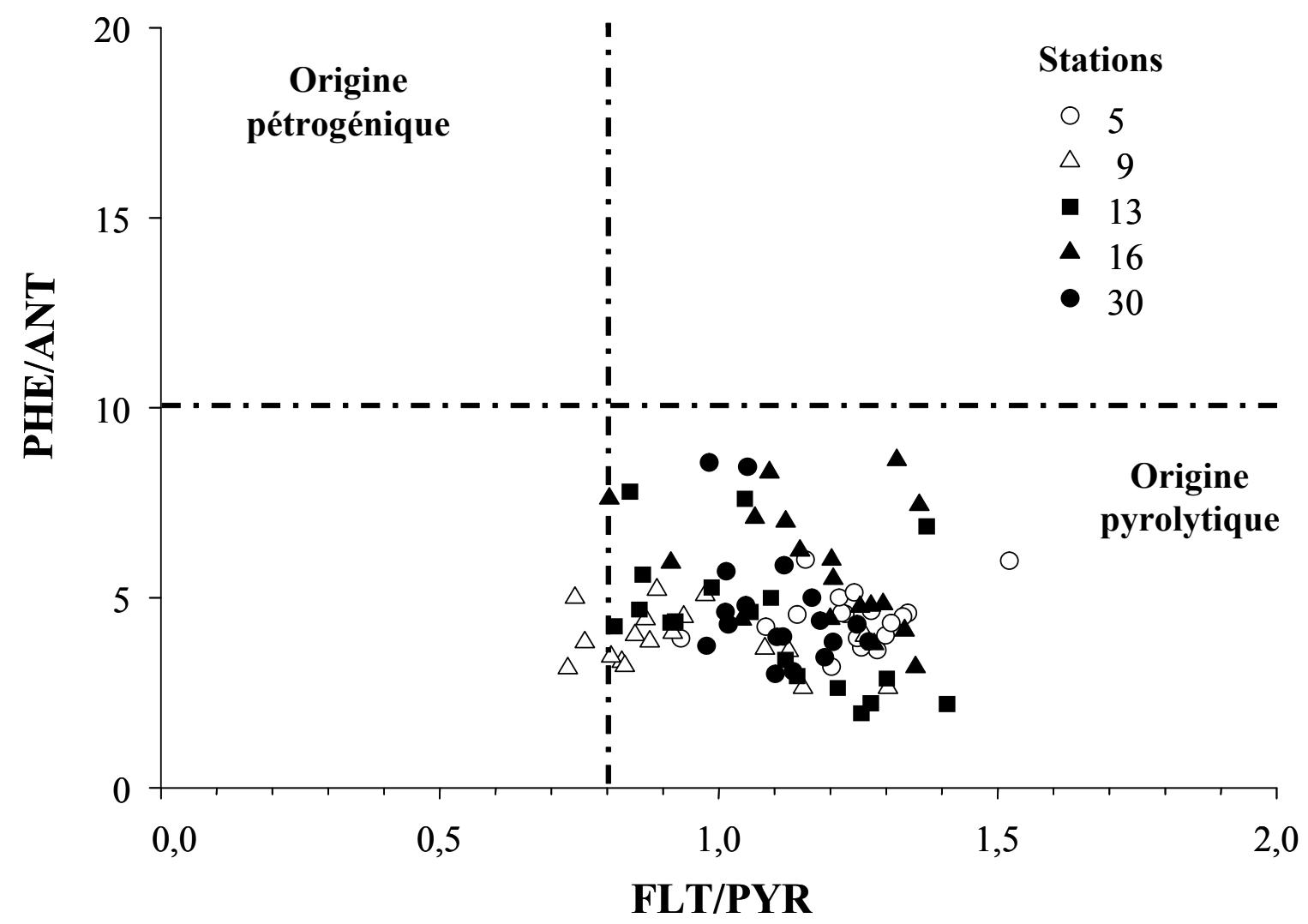

Figure 6. Relation entre le rapport fluorenthène/pyrène (FLT/PYR) et le rapport phenanthrène/anthracène (PHE/ANT) pour toutes les carottes et tous les sous-échantillons analysés dans le fjord du Saguenay en 2001 et 2002.

Relationship between fluoranthenelpyrene ratio (FLT/PYR) and phenanthrenelanthracene ratio (PHE/ANT) for all cores and all depth samples analyses from the Saguenay Fjord in 2001 and 2002. 
ainsi que quelques métabolites dans les tissus de quelques invertébrés. Même si les composés à 4 cycles dominent largement les sédiments de surface, les résultats obtenus pour les organismes benthiques et épi-benthiques échantillonnés en zone profonde montrent une prédominance des HAP légers à 2 et 3 cycles. Parmi les espèces échantillonnées, les polychètes (Nereis sp.) de la baie des Ha! Ha! montrent la plus forte bioaccumulation avec des concentrations de HAP totaux de $118 \mathrm{ng}^{\circ} \mathrm{g}^{-1}$ (St. 13), and $145 \mathrm{ng} \bullet^{-1}$ (St. 9-13) (Figure 7A). Des données similaires sont observées dans les crevettes échantillonnées aussi dans la baie des Ha! Ha! (100 ng•g ${ }^{-1}$; St. 7-9) (Figure 7B). Ces résultats pourraient apparaître contradictoires par rapport à la composition des sédiments de surface du fjord mais il faut voir que la bioaccumulation des HAP dans les organismes n'est pas en relation directe avec la composition des HAP d'un sédiment ou de particules en suspension. La bioaccumulation de composés organiques dépend d'abord de la biodisponibilité et de la bioaccessibilité de ces composés (TER LAAK et al., 2006). De nombreux travaux ont montré que la bioaccumulation des HAP chez les invertébrés était surtout liée à la portion libre du composé considéré et non à sa concentration totale dans le substrat (CORNELISSEN et al., 2006; MA et al., 1998). La détermination du BSAF (Benthos to Sediment Accumulation Factor) pour un sédiment donné est fonction de la concentration dissoute du HAP et de la quantité de carbone organique total se trouvant dans les sédiments (GRANBERG et SELCK, 2007). Comme les HAP à 2 ou 3 cycles sont beaucoup plus solubles que les composés lourds, leur facteur de bioaccumulation (BSAF) est beaucoup plus élevé que celui du benzo(a)pyrène, par exemple. Les données de la figure $7 \mathrm{~A}$ reflètent directement cet état de fait. L'exception semble être le chrysène, un composé à 4 cycles qui s'accumule dans les vers mais pas dans les crevettes. La raison de ce comportement particulier nous est inconnue pour l'instant.

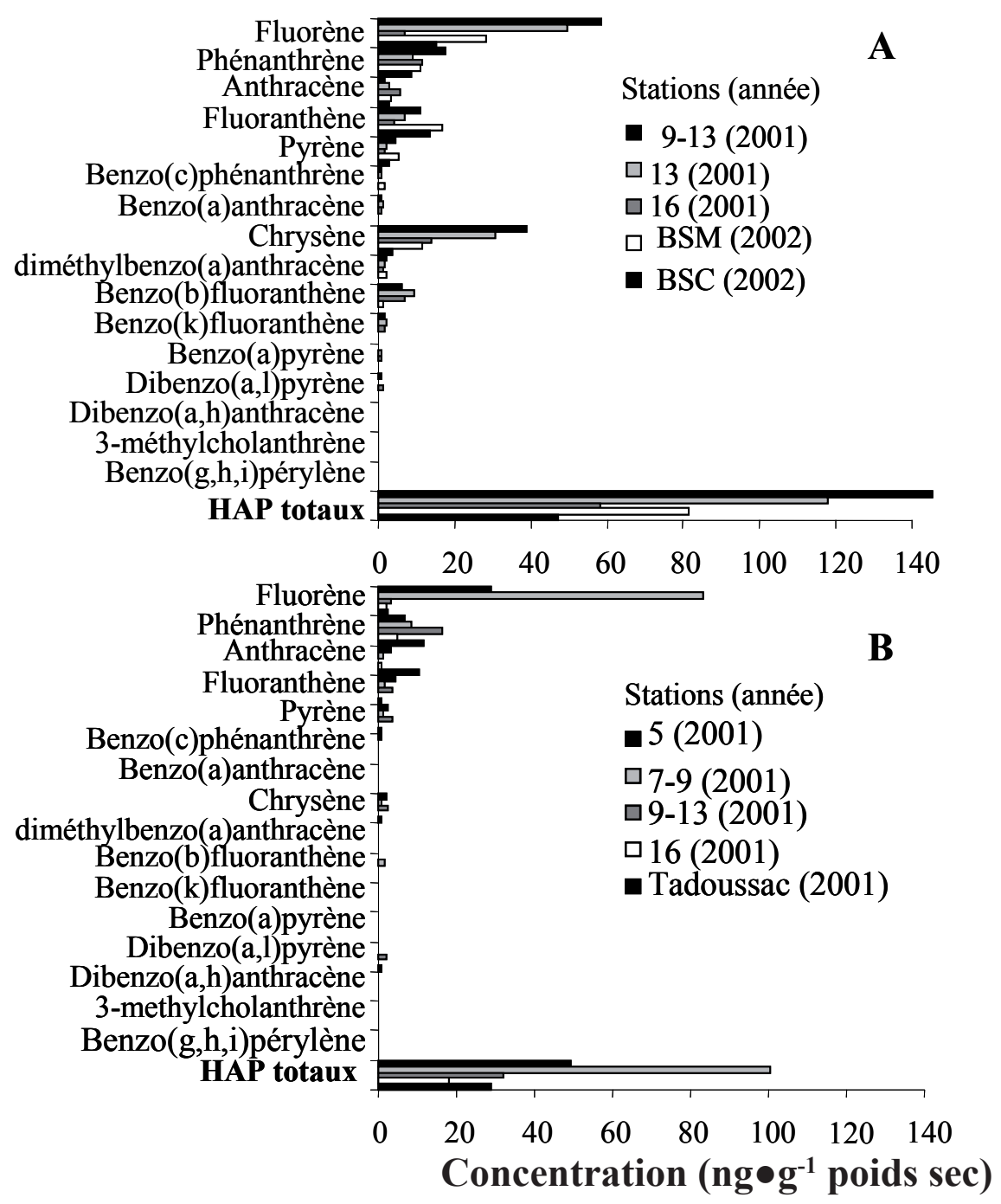

Figure 7. Distribution des HAP individuels et totaux (ng•g $\left.{ }^{-1}\right)$ dans les vers (A) et les crevettes (B) échantillonnés dans le fjord du Saguenay en 2003.

Distribution of individual and total PAHs (ng. $\left.g^{-1}\right)$ in worms (A), and shrimps (B) sampled in the Saguenay Fjord in 2003. 
Les moules et les myes échantillonnées dans la zone de battement de la marée de la baie Sainte-Marguerite et de la baie Sainte-Catherine, toutes deux proches de l'embouchure du fjord sur le Saint-Laurent, montrent aussi une bioaccumulation des HAP légers par rapport aux plus lourds (Tableau 3). Les HAP totaux sont significativement $(\mathrm{p}<0,05)$ plus concentrés

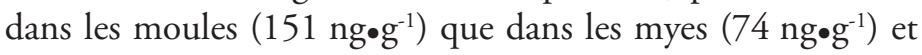

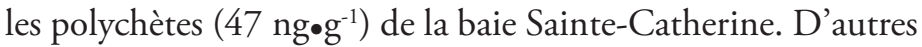
travaux ont montré que les HAP sont souvent accumulés à des niveaux plus élevés dans les moules que dans les myes (OROS et ROSS, 2005; WHITE et al., 1997).

\subsection{Présence des métabolites des HAP}

Il est maintenant clairement établi que la grande majorité des organismes terrestres et aquatiques, incluant les invertébrés benthiques, sont en mesure de métaboliser les HAP et de bioaccumuler les métabolites (DRISCOLLet McELROY, 1996; STROOMBERG et al., 2004). L'utilisation des métabolites comme indicateurs d'exposition aux hydrocarbures pétroliers a aussi été démontrée (DA SILVA et al., 2006), cependant encore peu de laboratoires analysent individuellement les hydroxy-PAHs dans les tissus biologiques. Plus récemment, JOHNSON-RESTREPO et al. (2008) ont démontré la présence de naphthalénols, phénanthrénols, pyrénols, benzo(a) pyrénols et de multiples autres composés hydroxylés dans la bile de poissons exposés en milieu naturel.

Nous avons recherché les métabolites dans les tissus de moules et des myes de la baie Sainte-Marguerite et la baie Sainte-Catherine (Tableau 3) et nous avons observé de faibles quantités de phénanthrénols et de pyrénols dans la majorité des tissus. Ces résultats préliminaires confirment la présence de métabolites des HAP dans les bivalves de la zone intertidale du fjord du Saguenay et sont un indice d'une exposition continue de ces organismes au phénanthrène et au pyrène. Cependant, on ne peut pas en tirer d'information de nature toxicologique pour le moment.

\section{CONCLUSION}

Nos résultats ne montrent pas de différences chimiques significatives entre la distribution des HAP particulaires échantillonnés en amont et en aval de la rivière Saguenay, même si on observe d'importantes variations saisonnières dans leur concentration. Par contre, d'importantes différences existent entre les HAP particulaires de la rivière et du ford montrant un changement drastique de leur composition

Tableau 3. HAP et hydroxy-HAP dans les tissus de la mye commune (Mya arenaria) et de la moule bleue (Mytilus edulis) échantillonnées en 2002. Les concentrations sont données en $\mathrm{ng}^{-1}{ }^{-1}$ poids sec. $\mathrm{BSC}=$ Baie-Sainte-Catherine; BSM = Baie-Sainte-Marguerite.

Table 3. PAHs and hydroxy-PAHs in tissues of soft shell clam (Mya arenaria) and blue mussel (Mytilus edulis) sampled in 2002. Concentrations are given in ng॰ ${ }^{-1}$ dry weight. BSC = Bay Sainte-Catherine; BSM = Bay Sainte-Marguerite.

\begin{tabular}{|c|c|c|c|}
\hline \multirow[t]{2}{*}{ HAP mesurés } & \multicolumn{2}{|c|}{ Station BSC } & \multirow{2}{*}{$\frac{\text { Station BSM }}{\text { Mye }}$} \\
\hline & Mye & Moule & \\
\hline Fluorène & 48,13 & 28,17 & 12,91 \\
\hline Phénanthrène & 6,20 & 34,08 & 12,83 \\
\hline Anthracène & 5,34 & 21,80 & 14,46 \\
\hline Fluoranthène & 5,02 & 53,91 & 11,75 \\
\hline Pyrène & 3,55 & 2,64 & 2,50 \\
\hline Benzo(c)phenanthrène & 0,99 & 4,09 & 0,74 \\
\hline Benzo(a)anthracène & 0,20 & 0,13 & 0,93 \\
\hline Chrysène & 1,58 & 3,03 & 5,29 \\
\hline 7,12-dimethylbenzo(a)anthracène & 0,44 & nd & 1,73 \\
\hline Benzo(b)fluoranthène & 1,85 & 3,00 & 4,23 \\
\hline Benzo(k)fluoranthène & 0,45 & 0,49 & 1,31 \\
\hline Benzo(a)pyrène & $\mathrm{nd}^{*}$ & 0,04 & 0,11 \\
\hline HAP totaux & 73,75 & 151,42 & 68,8 \\
\hline Phénanthrénols & nd & 0,92 & 8,45 \\
\hline Pyrénols & 0,27 & 0,22 & 0,21 \\
\hline Hydroxy-HAP totaux & 0,27 & 1,14 & 8,66 \\
\hline
\end{tabular}

* nd $=$ non détecté 
en passant des composés légers de la section fluviale à des composés lourds pour la section marine. La MPS de la rivière apparaît de huit à 34 fois plus concentrée en HAP que celle du fjord mais la rivière transporte relativement peu de HAP en matière d'apports globaux au fjord à cause de sa faible charge en MPS. Il n'y a pas d'évidence d'une contribution importante de l'industrie lourde, et notamment des alumineries, au bilan de masse des HAP dans la rivière Saguenay, réalisé au début des années 2000 contrairement aux données rapportées pour les années 1950 à 1980.

Nos nouvelles données ont permis de réévaluer la distribution spatiale des HAP dans le Saguenay et de discuter des sources possibles 20 ans après la première évaluation publiée par MARTEL et al. (1987). Nos résultats de 2002 montrent une réduction majeure des HAP dans tous les secteurs géographiques du ford et les profils des carottes confirment le lent processus d'enfouissement des sédiments contaminés par de nouveaux sédiments ayant des concentrations en HAP totaux entre 200 et $400 \mathrm{ng}_{\bullet} \mathrm{g}^{-1}$, ce qui est en dessous du seuil

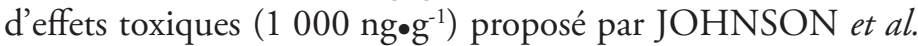
(2002). Les bivalves en zone littorale sont clairement exposés aux hydrocarbures pétroliers, tout au moins durant la période estivale. La source peut être aussi bien hydrique et pétrogénique (déversements accidentels de carburants et eaux usées des municipalités) qu'atmosphérique via les tuyaux d'échappement des voitures et camions (TAVARES et al., 2004).

\section{REMERCIEMENTS}

Ces travaux ont été supportés financièrement par le CRSNG (Conseil de recherche en sciences naturelles et en génie du Canada) via son programme des subventions stratégiques, Alcan Ltée et la Chaire de recherche du Canada en écotoxicologie moléculaire (E.P). Ce travail est une contribution de Québec-Océan (FQRNT).

\section{RÉFÉRENCES}

BATES T.S., P.P. MURPHY, H.C. CURL et R.A. FEELY (1987). Hydrocarbon distribution and transport in an urban estuary. Environ. Sci. Technol., 21, 193-198.

BAUMARD P., H. BUDZINSKI et P. GARRIGUES (1998). Polycyclic aromatic hydrocarbons in sediments and mussels of the Western Mediterranean Sea. Environ. Toxicol. Chem., $17,765-776$.
BRION D. et E. PELLETIER (2005). Modelling PAHs adsorption and sequestration in freshwater and marine sediments. Chemosphere, 61, 867-876.

COUILLARD, C.M. (2008). Utilisation des poissons pour évaluer les effets biologiques des contaminants dans l'estuaire du Saint-Laurent et du fjord du Saguenay. Rev. Sci. Eau, (ce numéro).

COUILLARD, C.M., B. LAPLATTE et É. PELLETIER (2009). A fish bioassay to evaluate the toxicity associated with the ingestion of benzo[a]pyrene-contaminated benthic prey. Environ. Toxicol. Chem., 28, 772-781.

CRE (1988). Les états généraux de l'environnement du Saguenayl Lac-Saint-Jean: état de la situation: pour notre avenir a tous. Conseil régional de concertation et de développement Saguenay-Lac-Saint-Jean-Chibougamau-Chapais, Jonquière, Québec.

DA SILVA D., J. BUZITIS, M.M. KRAHN, M.C. BICEGO et A.M.S. PIRES-VANIN (2006). Metabolites in bile of fish from Sao Sebastiao Channel, Sao Paulo, Brazil as biomarkers of exposure to petrogenic polycyclic aromatic compounds. Mar. Pollut. Bull., 52, 175-183.

DOONG R.A. et Y.T. LIN (2004). Characterization and distribution of polycyclic aromatic hydrocarbon contamination in surface sediment and water from Gaoping River, Taiwan. Water Res., 38,1733-1744.

DRAINVILLE, G. (1968). Le fjord du Saguenay: Contribution à l'océanographie. Natur. Can., 95, 809-855.

DRISCOLL S.K. et A.E. MCELROY (1996). Bioaccumulation and metabolism of benzo(a)pyrene in three species of polychaete worms. Environ. Toxicol. Chem., 15, 1401-1410.

FERNANDES M.B., M.A. SICRE, A. BOIREAU et J. TRONCZYNSKI (1997). Polyaromatic hydrocarbons (PAH) distributions in the Seine River and its Estuary. Mar. Pollut. Bull., 34, 857-867.

FERNANDES M.B., M.A. SICRE, I. BROYELLE, A. LORRE et B. PONT (1999). Contamination by polycyclic aromatic hydrocarbons (PAHs) in French and European rivers. Hydrobiologia, 410, 343-348.

FORTIN G.R. et M. PELLETIER (1995). Synthèse des connaissances sur les aspects physiques et chimiques de l'eau et des sédiments de Saguenay. Zones d'intervention prioritaire 22 et 23. Environnement Canada, région du Québec, Conservation de l'environnement, Centre Saint-Laurent, Montréal, Québec, 57 p. 
JOHNSON L.L., T.K. COLLIER et J.E. STEIN (2002). An analysis in support of sediment quality thresholds for polyclic aromatic hydrocarbons (PAHs) to protect estuarine fish. Aquat. Conser.: Mar. Freshwater Ecol., 12, 517-538.

JOHNSON-RESTREPO B., J. OLIVERO-VERBEL, S. LU, J. GUETTE-FERNANDEZ, R. BALDIRIS-AVILA, I. O'BYRNE-HOYOS, K.M. ALDOUS, R. ADDINK et K. KANNAN (2008). Polyaromatic aromatic hydrocarbons and their hydroxylated metabolites in fish bile and sediments from coastal waters of Colombia. Environ. Pollut., 151, 452-459.

GRANBERG M.E. et H. SELCK (2007). Effects of sediments organic matter quality on bioaccumulation, degradation and distribution of pyrene in two macrofaunal species and their surrounding sediment. Mar. Environ. Res., 64, 313-335.

GSCHWEND P.M. et R.A. HITES (1981). Fluxes of polycyclic aromatic hydrocarbons to marine and lacustrine sediments in the northeastern US. Geochim. Cosmochim. Acta, 45, 2359-2367.

KHIM J.S., K.T. LEE, K. KANNAN, D.L. VILLENEUVE, J.P. GIESY et C.H. KOH (2001). Trace organic contaminants in sediment and water from Ulsan Bay and its vicinity, Korea. Arch. Environ. Contam. Toxicol., 40,141-150.

KRANTZBERG, G. (1985). The influence of bioturbation on physical, chemical and biological parameters in aquatic environments: A review. Environ. Pollut. (A), 39, 99-122.

LECLERC A., M.J. GAGNON et A. MAMI (1986). Compaction and movement of interstitial water in bottom sediments of the Saguenay Fjord, Quebec, Canada. Sediment. Geol., 46, 213-230.

LEI L., A.P. KHODADOUST, M.T. SUIDON et H.H. TABAK (2005). Biodegradation of sediment-bound $\mathrm{PAHs}$ in field-contaminated sediment. Water Res., 39, 349-361.

LUN R., K. LEE, L. DE MARCO, C. NALEWAJKO et D. MACKAY (1998). A model of polycyclic aromatic hydrocarbons in the Saguenay Fjord, Canada. Environ. Toxicol. Chem., 17, 333-341.

MA W.-C., A. VAN KLEUVEN, J. IMMERZEEL, et P. GERT-JAN DE MAAGD (1998). Bioaccumulation of polycyclic aromatic hydrocarbons by earthworms: assessment of equilibrium partitioning theory in in-situ studies and water experiments. Environ. Toxicol., 17,1730-1737.
MACKAY D., W.Y. SHIU et K.C. MA (1992). Illustrated handbook of physical-chemical properties and environmental fate for organic chemicals. Volume II: Polycyclic aromatic hydrocarbons, polychlorinated dioxins, and dibenzofurans. Lewis Publishers, Chelsea, Michigan, USA, 597 p.

MANOLI E., C. SAMARA, I. KONSTANTINOU et T. ALBANIS (2000). Polycyclic aromatic hydrocarbons in the bulk precipitation and surface water of Northern Greece. Chemosphere, 41, 1845-1855.

MARTEL L., J.M. GAGNON, R. MASSÉ, A. LECLERC et L. TREMBLAY (1986). Polycyclic aromatic hydrocarbons in sediments from the Saguenay Fjord, Canada. Bull. Environ. Contam. Toxicol., 37, 133-140

MARTEL L., M.J. GAGNON, R. MASSÉ et A. LECLERC (1987). The spatio-temporal variations and fluxes of polycyclic aromatic hydrocarbons in the sediments of the Saguenay Fjord, Quebec, Canada. Water Res., 21, 699-707.

MARTINEAU D., K. LEMBERGER, A. DALLAIRE, P. LABELLE, T.P. LIPSCOMB, P. MICHEL et I. MIKAELIAN (2002). Cancer in wildlife, a case study: beluga from the St. Lawrence Estuary, Québec, Canada. Environ. Health Persp., 110, 1-8.

MASKAOUI K., J.L. ZHOU, H.S. HONG et Z.L. ZHANG (2002). Contamination by polycyclic aromatic hydrocarbons in the Jiulong River Estuary and Western Xiamen Sea, China. Environ. Pollut., 118, 109-122.

OUELLET S. (1990). Etude des hydrocarbures aliphatiques et aromatiques polycycliques (HAP) dans les sédiments du ford du Saguenay. Mémoire de maitrise en océanographie, Université du Québec à Rimouski, Rimouski, Québec, Canada, M194, 149 p.

OROS D.R. et J.R.M. ROSS (2005). Polycyclic aromatic hydrocarbons in bivalves from the San Francisco Estuary: Spatial distributions, temporal trends, and sources (1993-2001). Mar. Environ. Res., 60, 466-488.

OROS, D.R., J.R.M. ROSS, R.B.SPIES et T. MUMLEY (2007). Polycyclic aromatic hydrocarbon (PAH) contamination in the San Francisco Bay: A 10-year retrospective of monitoring in an urbanized estuary. Environ. Sci., 105, 101-118.

PELLETIER É., B. DEFLANDRE, C. NOZAIS, G. TITA, G. DESROSIERS, J.-P. GAGNÉ et A. MUCCI (1999). 
Crue éclair de juillet 1996 dans la région du Saguenay, Québec. 2. Impacts sur les sédiments et le biote de la baie des Ha! Ha! et du fjord du Saguenay. Can. J. Fish. Aquat. Sci., 56, 2136-2147.

PELLETIER É, G. DESROSIERS, J. LOCAT, A. MUCCI et H. TREMBLAY (2003). The origin and behavior of a flood capping layer deposited on contaminated sediments of the Saguenay Fjord, Quebec, Dans: Contaminated Sediments: Characterization, Evaluation, Mitigation/ Restoration, and Management Strategy Performance. LOCAT J., R. GALVEZ-CLOUTIER, R.C. CHANEY, et K.R. DEMARS (Éditeurs), STM STP 1442, ASTM International, West Conshohocken, PA, USA

PEREIRA W.E., F.D. HOSTETTLER et J.B. RAPP (1996). Distributions and fate of chlorinated pesticides, biomarkers and polycyclic aromatic hydrocarbons in sediments along a contamination gradient from a point-source in San Francisco Bay, California. Mar. Environ. Res., 41, 299-314.

READMAN J.W., R.F.C. MANTOURA et M.M. RHEAD (1987). A record of polycyclic aromatic hydrocarbon (PAH) pollution obtained from accreting sediments of the Tamar estuary, UK: evidence for non-equilibrium behaviour of PAH. Sci. Total Environ., 66, 73-94.

SMITH J.N. et E.M. LEVY (1990).Geochronology for polycyclic aromatic hydrocarbon contamination in sediment of the Saguenay Fjord. Environ. Sci. Technol., 24, 874-879.

STARK A., J.R. ABROJANO JR, J. HELLOU et J.L. METCALF-SMITH (2003). Molecular and isotopic characterization of polycyclic aromatic hydrocarbon distribution and sources at the international segment of the St. Lawrence River. Org. Geochem., 34, 225-237.

STRAIF K., R. BAAN, Y. GROSSE, B. SECRETAN, F. EL GHISSASSI et V. COGLIANO (2005). Carcinogenicity of polycyclic aromatic hydrocarbons. Lancet Oncol. J., 6, 931-932.

STROOMBERG G.J., H. ZAPPEY, R.J.C.A. STEEN, C.A.M. CAN GESTEL, F. ARIESE, N.H. VELTHORST et N.M. VAN STRAALEN (2004). PAH biotransformation in terrestrial invertebrates - a new phase II metabolite in isopods and springtails. Comp. Biochem. Physiol., C, 138, 129-137.

TAVARES Jr M., J.P. PINTO, I.S. SCARMINIO, M.C. SOLCI et A.L. SOUZA (2004). Emission of polycyclic hydrocarbons from diesel engine in a bus station, Londrina, Brazil. Atmos. Environ., 38, 5039-5044.
TELLI-KARAKOÇ F., L. TOLUN, B. HENKELMANN, C. KLIMM, O. OKAY et K.W. SCHRAMM (2002). Polycyclic aromatic hydrocarbons (PAHs) and polychlorinated biphenyls (PCBs) distribution in the Bay of Marmara Sea: İzmit Bay. Environ. Pollut., 119, 383-397.

TER LAAK T.L., A. BARENDREGT et J.L.P. HERMENS (2006). Freely dissolved pore water concentrations and sorption coefficients of PAHs in spiked, aged, and fieldcontaminated soils. Environ. Sci. Technol., 40, 2184-2190.

THIBODEAUX L.J., K.T. VALSARAJ et D.D. REIBLE (2001). Bioturbation-driven transport of hydrophobic organic contaminants from bed sediment. Environ. Eng. Sci., 18, 215-233.

VAN DER OOST R., J. BEYER et V.N.P. ERMEULEN (2003). Fish bioaccumulation and biomarkers in environmental risk assessment: a review. Environ. Toxicol. Pharmacol., 13, 57-149.

VARANASI U. et J.E. STEIN (1991). Disposition of xenobiotic chemicals and metabolites in marine organisms. Environ. Health Perspec., 90, 93-100.

VIGLINO L., É. PELLETIER et R. ST-LOUIS (2004). Highly persistent butyltins in northern marine sediments: a longterm for the Saguenay Fjord (Canada). Environ. Toxicol. Chem., 23, 2673-2681.

WHITE P.A., C. BLAISE et J.B. RASMUSSEN (1997). Detection of genotoxic substances in bivalve mollusks from the Saguenay Fjord (Canada), using the SOS chromotest. Mutation Res., 392, 277-300.

YUNKER M.B., S.M. BACKUS, E. GRAF PANNATIER, D.S. JEFFRIES et R.W. MACDONALD (2002). Sources and significances of alkanes and PAH hydrocarbons in Canadian Arctic rivers. Estuar. Coast. Shelf Sci., 55, 1-31.

ZHOU J.L. et S.J. ROWLAND (1997). Evaluation of the interactions between hydrophobic organic pollutants and suspended particles in estuarine waters. Water Res., 31, 1708-1718. 